\title{
EVALUATION OF SIDE RESISTANCE CAPACITY FOR DRILLED SHAFTS
}

Yit-Jin Chen

Department of Civil Engineering, Chung Yuan Christian University ChungLi 32023, Taiwan, R.O.C., ycj@cycu.edu.tw

Shiu-Shin Lin

Department of Civil Engineering, Chung Yuan Christian University ChungLi 32023, Taiwan, R.O.C.

\section{Hsin-Wen Chang}

Department of Civil Engineering, Chung Yuan Christian University ChungLi 32023, Taiwan, R.O.C.

Maria Cecilia Marcos

Department of Civil Engineering, Chung Yuan Christian University ChungLi 32023, Taiwan, R.O.C.

Follow this and additional works at: https://jmstt.ntou.edu.tw/journal

Part of the Civil and Environmental Engineering Commons

\section{Recommended Citation}

Chen, Yit-Jin; Lin, Shiu-Shin; Chang, Hsin-Wen; and Marcos, Maria Cecilia (2011) "EVALUATION OF SIDE RESISTANCE CAPACITY FOR DRILLED SHAFTS," Journal of Marine Science and Technology. Vol. 19: Iss. 2, Article 13.

DOI: $10.51400 / 2709-6998.2156$

Available at: https://jmstt.ntou.edu.tw/journal/vol19/iss2/13

This Research Article is brought to you for free and open access by Journal of Marine Science and Technology. It has been accepted for inclusion in Journal of Marine Science and Technology by an authorized editor of Journal of Marine Science and Technology. 


\title{
EVALUATION OF SIDE RESISTANCE CAPACITY FOR DRILLED SHAFTS
}

\author{
Yit-Jin Chen*, Shiu-Shin Lin*, Hsin-Wen Chang*, and Maria Cecilia Marcos*
}

Key words: drilled shaft, load test, side resistance, case history.

\begin{abstract}
This paper presents an extensive evaluation of axial side resistance of drilled shaft foundations. A wide variety of load test data are used and these data are divided into drained and undrained databases. Representative analytical models, including alpha $(\alpha)$, beta $(\beta)$, and lambda $(\lambda)$ methods, are examined in detail using both measured and predicted results to assess their relative merits for the drilled shaft design. Based on these analyses, the undrained shear strength $\left(\alpha-s_{u}\right)$ correlations have exhibited better statistics for undrained side resistance prediction, and the undrained strength ratio ( $\alpha$-USR) correlations can be adopted as an alternative analytical method, especially in the case of smaller $s_{u}$. For $\beta$ method, the stress factors $\left(\mathrm{K} / \mathrm{K}_{\mathrm{o}}\right)$ are developed from the back-analysis of field load tests. However, the $\beta$ method has presented more varying results for short shafts in both drained and undrained loading. Among all analytical methods, the $\lambda$ method is relatively the less reliable prediction model. Specific design recommendations for side resistance analysis of drilled shaft are suggested.
\end{abstract}

\section{INTRODUCTION}

Drilled shafts (also called cast-in-place piles, drilled piers or bored piles) are frequently used as foundation for modern high-rise buildings, bridges, electrical transmission line structures, etc. Side resistance is an important source of drilled shaft capacity under axial loading, especially when the shaft is under the condition of uplift loading or considerably larger depth. Researches about this subject still have progressed during the past five decades. Based on soil conditions, the methods for analyzing side resistance are of two types: total stress analysis and effective stress analysis. These analytical methods can be further specified into the alpha $(\alpha)$, beta $(\beta)$, and lambda $(\lambda)$ methods. Table 1 lists the equations and the related factors for each method.

The $\alpha$ method is a conventional total stress analysis for the

Paper submitted 11/30/09; revised 03/27/10; accepted 04/04/10. Author for correspondence: Yit-Jin Chen (e-mail: ycj@cycu.edu.tw).

*Department of Civil Engineering, Chung Yuan Christian University ChungLi 32023, Taiwan, R.O.C. side resistance of drilled shaft foundations in cohesive soils. The side resistance capacity is related to the average soil undrained shear strength $\left(\mathrm{s}_{\mathrm{u}}\right)$ by an empirical coefficient denoted as $\alpha$, which is the adhesion factor. The original $\alpha$ [14] was based on empirical correlations of mean $\mathrm{s}_{\mathrm{u}}$ over the foundation depth, using primarily driven pile data. The research from Stas and Kulhawy [13] showed that $\alpha$ method is meaningful for drilled shafts. They developed the correlation of $\alpha$ versus $\mathrm{s}_{\mathrm{u}}$ for the drilled shaft design, however, the $\mathrm{s}_{\mathrm{u}}$ values in their analysis were taken from random test types, as shown in Fig. 1.

Moreover, several researchers $[7,11,12]$ also demonstrated that $\alpha$ is complexly related to other soil parameters such as the mean effective overburden stress $\left(\bar{\sigma}_{\mathrm{vm}}\right)$, the overconsolidation ratio (OCR), and the effective stress friction angle $(\bar{\phi})$. With these suggestions, Goh et al. [4] further carried out parametric studies using the trained neural network model and proposed that $\bar{\sigma}_{\mathrm{vm}}$ can directly or indirectly influence $\alpha$ in designing drilled shafts.

The $\beta$ method is an effective stress analysis which considers the frictional resistance for the soil-shaft interface. In this method, the side resistance is a function of horizontal effective stress $\left(\bar{\sigma}_{\text {ho }}\right)$, effective stress friction angle $(\delta)$ for the soil-shaft interface, and shaft geometry. Kulhawy et al. [9] examined the available load test data and presented that the stress factor $\left(\mathrm{K} / \mathrm{K}_{\mathrm{o}}\right)$ is generally less than 1 and dependent on the construction method and its influence on the in-situ stress. They also suggested that the ratio of interface friction angle $(\delta)$ to soil friction angle $(\bar{\phi})$ is equal to 1 for drilled shafts.

Finally, the lambda $(\lambda)$ method is a combination of total and effective stress analyses that can be used for cohesive soils. In this method, the side resistance is related to $s_{u}$ and $\bar{\sigma}_{v m}$ by an empirical factor $\lambda$. The original empirical factor $\lambda$ [15] was developed based on the database of driven pile data and is a function of the total depth of the pile. However, no further effort has been done in detail to examine its validity in the design of drilled shafts.

Although some of these analytical methods have been examined previously, it is reasonable to completely reassess these methods for the side resistance analysis of drilled shaft design, because new approaches have been developed, and more consistent procedures for assessing interpreted failure 
Table 1. Analysis equations of side resistance for drilled shafts.

\begin{tabular}{|c|c|c|c|}
\hline Method & Equation $^{\mathrm{a}}$ & & Definition of factors \\
\hline$\alpha$ & $\mathrm{Q}_{\mathrm{s}}(\alpha)=\pi \mathrm{B} \sum_{\mathrm{n}=1}^{\mathrm{N}} \alpha_{\mathrm{n}} \mathrm{s}_{\mathrm{un}} \mathrm{t}_{\mathrm{n}}$ & (1) & $\begin{array}{l}\alpha \text { : empirical adhesion factor } \\
\mathrm{s}_{\mathrm{u}}: \text { undrained shear strength }\end{array}$ \\
\hline$\beta$ & $\mathrm{Q}_{\mathrm{s}}(\beta)=\pi \mathrm{B}\left(\frac{\mathrm{K}}{\mathrm{K}_{\mathrm{o}}}\right) \sum_{\mathrm{n}=1}^{\mathrm{N}} \bar{\sigma}_{\mathrm{vn}} \mathrm{K}_{\mathrm{on}} \tan \left[\bar{\phi}_{\mathrm{n}} \cdot \frac{\delta}{\bar{\phi}}\right] \mathrm{t}_{\mathrm{n}}$ & (2) & $\begin{array}{l}\mathrm{K}: \text { coefficient of horizontal soil stress } \\
\frac{\mathrm{K}_{\mathrm{o}}}{\bar{\sigma}_{\mathrm{v}}} \text { : in-situ } \mathrm{K} \\
\bar{\phi}: \text { vertical effective stress } \\
\delta \text { : effective stress friction angle } \\
\beta=\mathrm{K} \tan \delta\end{array}$ \\
\hline$\lambda$ & $\mathrm{Q}_{\mathrm{s}}(\lambda)=\pi \mathrm{B} \sum_{\mathrm{n}=1}^{\mathrm{N}} \lambda_{\mathrm{n}}\left[\bar{\sigma}_{\mathrm{vn}}+2 \mathrm{~s}_{\mathrm{un}}\right] \mathrm{t}_{\mathrm{n}}$ & (3) & $\lambda$ : empirical factor \\
\hline
\end{tabular}

${ }^{\mathrm{a}} \mathrm{Q}_{\mathrm{s}}=$ capacity of side resistance; $\mathrm{B}=$ shaft diameter; $\mathrm{N}=$ number of soil layers; $\mathrm{t}=$ thickness.

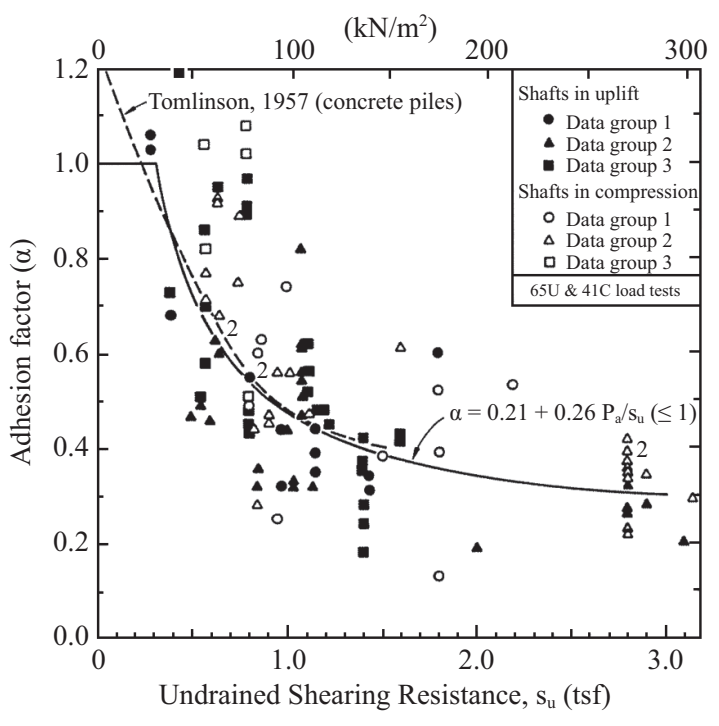

Fig. 1. $\alpha-s_{u}$ correlation [13].

load have been improved, additionally, numerous updated load test data have existed since those earlier studies. In this paper, a broad database is used to assess the relative merits and suitability of each analytical model using the most updated data and approaches. The results are compared statistically and graphically. After which, specific design recommendations for the use of side resistance in drilled shaft design are presented.

\section{DATABASE OF LOAD TESTS}

The database developed for this study is consisted of 222 field axial load tests conducted at 105 sites with a wide variety of soil profiles. All load test data were collected from published literature or load test reports. Based on primarily dominant soil conditions along the shaft length, axial load test data are grouped into drained or undrained loading. Among these load test data, 74 tests at 40 sites are grouped into drained loading and 148 tests at 65 sites are grouped into undrained loading. Load tests are divided into two groups, each based on the overall data quality, completing a total of eight-sub groups as follows: Group 1 in compression (denoted DC1 and UC1 for drained and undrained soils, respectively), Group 2 in compression (DC2 and UC2), Group 1 in uplift (DU1 and UU1), and Group 2 in uplift (DU2 and UU2). Group 1 includes those cases in which the complete load-displacement curve and geotechnical parameters were reported. Group 2 consists of all remaining cases, such as the groundwater table was not reported, the load test was stopped before interpreted failure load, or the geotechnical parameters were not measured over the entire depth, and therefore they have to be inferred from other tests. All of the selected tests were conducted on straight-sided drilled shafts. Based on the case history descriptions, it appears that the shaft constructions and test performances were of high quality. Consequently, these field data should reflect real situations and the results of the analyses could be representative for subsequent applications in engineering practice.

Tables 2 and 3 show the interpreted parameters and result of the analyses for the load tests with drained and undrained soils, respectively. The values of $\alpha, \beta_{\mathrm{m}}$, and $\lambda$ are back-calculated using Eqs. (1)-(3) from the field load test results. The $\mathrm{L}_{1}-\mathrm{L}_{2}$ method $[5,6]$, which is a graphical construction method, is adopted to define the capacity in both uplift and compression. As shown in Fig. 2, the load-displacement curves can generally be simplified into three distinct regions: initial linear, curve transition, and final linear. Point $\mathrm{L}_{1}$ (elastic limit) corresponds to the load $\left(\mathrm{Q}_{\mathrm{L} 1}\right)$ and butt displacement $\left(\rho_{\mathrm{L} 1}\right)$ at the end of the initial linear region, while $\mathrm{L}_{2}$ (failure threshold) corresponds to the load $\left(\mathrm{Q}_{\mathrm{L} 2}\right)$ and butt displacement $\left(\rho_{\mathrm{L} 2}\right)$ at the initiation of the final linear region. $\mathrm{Q}_{\mathrm{L} 2}$ is defined as the "interpreted failure load or capacity" because beyond $\mathrm{Q}_{\mathrm{L} 2}$, a small increase in load gives a significant increase in displacement. The updated research studies by Chen et al. [1] and Chen and Fang [2] defined that $\mathrm{Q}_{\mathrm{L} 2}$ occurs on average, at $10.6 \mathrm{~mm}$ (for drained loading) and $12.1 \mathrm{~mm}$ (for undrained loading) under uplift loading, while it occurs at $4 \% \mathrm{~B}$ for both drained and undrained compression 
Table 2. Summary of analysis data for drained loading.

\begin{tabular}{|c|c|c|c|c|c|c|c|c|c|c|c|c|c|}
\hline $\begin{array}{c}\text { Site/Case } \\
\text { No. }\end{array}$ & $\begin{array}{c}\mathrm{Q}_{\mathrm{s}} \\
(\mathrm{kN})\end{array}$ & $\begin{array}{c}\bar{\sigma}_{\mathrm{vm}}{ }^{\mathrm{a}} \\
\left(\mathrm{kN} / \mathrm{m}^{2}\right)\end{array}$ & $\begin{array}{c}\bar{\phi}_{\mathrm{tc}}{ }^{\mathrm{b}} \\
(\mathrm{deg})\end{array}$ & $\mathrm{K}_{\mathrm{o}}{ }^{\mathrm{c}}$ & $\beta_{\mathrm{m}}{ }^{\mathrm{d}}$ & $\beta_{p}{ }^{e}$ & $\begin{array}{c}\text { Site/case } \\
\text { No. }\end{array}$ & $\begin{array}{c}\mathrm{Q}_{\mathrm{s}} \\
(\mathrm{kN})\end{array}$ & $\begin{array}{c}\bar{\sigma}_{\mathrm{vm}}{ }^{\mathrm{a}} \\
\left(\mathrm{kN} / \mathrm{m}^{2}\right)\end{array}$ & $\begin{array}{c}\bar{\phi}_{\mathrm{tc}}{ }^{\mathrm{b}} \\
(\mathrm{deg})\end{array}$ & $\mathrm{K}_{\mathrm{o}}^{\mathrm{c}}$ & $\beta_{\mathrm{m}}{ }^{\mathrm{d}}$ & $\beta_{\mathrm{p}}{ }^{\mathrm{e}}$ \\
\hline DU1 & 334 & 27 & 36 & 1.4 & 1.42 & 1.05 & DU21-2 & 52 & 42 & 36 & 1.3 & 0.97 & 0.59 \\
\hline DU2 & 58 & 15 & 35 & 1.0 & 0.76 & 0.72 & DU21-3 & 74 & 76 & 35 & 0.9 & 0.63 & 1.02 \\
\hline DU3 & 362 & 14 & 36 & 3.1 & 2.97 & 2.43 & DU21-4 & 90 & 76 & 35 & 0.9 & 0.63 & 0.70 \\
\hline DU4 & 484 & 42 & 35 & 1.1 & 0.93 & 0.79 & DC1 & 488 & 42 & 33 & 0.9 & 0.60 & 1.56 \\
\hline DU5-1 & 24 & 13 & 36 & 3.1 & 1.36 & 1.64 & DC2 & 978 & 42 & 36 & 2.1 & 1.57 & 0.69 \\
\hline DU5-2 & 68 & 19 & 37 & 2.5 & 1.50 & 1.38 & DC3-1 & 1779 & 106 & 36 & 0.5 & 0.37 & 1.34 \\
\hline DU5-3 & 159 & 25 & 37 & 2.0 & 1.78 & 1.10 & DC3-2 & 3202 & 99 & 37 & 0.7 & 0.54 & 1.61 \\
\hline DU6-1 & 650 & 32 & 36 & 2.1 & 0.94 & 1.11 & DC4 & 548 & 50 & 30 & 1.8 & 1.07 & 0.93 \\
\hline DU6-2 & 650 & 32 & 36 & 2.1 & 0.94 & 1.11 & DC5 & 1246 & 89 & 40 & 0.8 & 0.49 & 0.96 \\
\hline DU7-1 & 390 & 42 & 44 & 1.0 & 0.66 & 0.99 & DC6 & 5907 & 96 & 34 & 0.7 & 0.44 & - \\
\hline DU7-2 & 498 & 42 & 44 & 1.0 & 0.77 & 0.99 & DC7 & 4270 & 86 & 38 & 1.1 & 0.89 & - \\
\hline DU8-1 & 261 & 56 & 35 & 0.4 & 0.32 & 0.31 & DC8-1 & 2755 & 89 & 40 & 0.7 & 0.59 & 1.00 \\
\hline DU8-2 & 278 & 56 & 35 & 0.4 & 0.28 & 0.31 & DC8-2 & 3528 & 89 & 40 & 0.7 & 0.59 & 0.97 \\
\hline DU9-1 & 1028 & 29 & 40 & 3.6 & 3.03 & 2.93 & DC9 & 144 & 50 & 35 & 1.1 & 0.75 & 1.02 \\
\hline DU9-2 & 1225 & 35 & 36 & 3.6 & 2.50 & 2.54 & DC10-1 & 1440 & 127 & 36 & 0.5 & 0.37 & 0.92 \\
\hline DU10-1 & 605 & 20 & 44 & - & 6.08 & - & DC10-2 & 1504 & 127 & 36 & 0.5 & 0.37 & 0.96 \\
\hline DU10-2 & 894 & 29 & 44 & - & 4.35 & - & DC10-3 & 1280 & 118 & 36 & 0.5 & 0.37 & 0.96 \\
\hline DU11-1 & 205 & 38 & 35 & - & 0.83 & - & DC10-4 & 1520 & 135 & 36 & 0.5 & 0.37 & 0.80 \\
\hline DU11-2 & 273 & 26 & 45 & - & 2.42 & - & DC10-5 & 1696 & 127 & 36 & 0.5 & 0.37 & 1.08 \\
\hline DU11-3 & 180 & 25 & 40 & - & 1.73 & - & DC11 & 4000 & 210 & 35 & 0.4 & 0.27 & 0.97 \\
\hline DU11-4 & 372 & 21 & 51 & - & 5.03 & - & DC12 & 9963 & 108 & 43 & 1.1 & 0.75 & 1.72 \\
\hline DU11-5 & 115 & 30 & 31 & - & 0.87 & - & DC13 & 1637 & 94 & - & - & - & - \\
\hline DU11-6 & 54 & 30 & 31 & - & 0.38 & - & DC14 & 2562 & 50 & 38 & 2.2 & 1.77 & 0.98 \\
\hline DU11-7 & 67 & 30 & 31 & - & 0.48 & - & DC15 & 6832 & 83 & 34 & - & - & - \\
\hline DU11-8 & 108 & 30 & 31 & - & 0.77 & - & DC16-1 & 4494 & 61 & - & - & - & - \\
\hline DU11-9 & 141 & 44 & 31 & - & 0.45 & - & DC16-2 & 2092 & 61 & & & - & - \\
\hline DU11-10 & 135 & 44 & 31 & - & 0.44 & - & DC17-1 & 5160 & 90 & - & - & - & - \\
\hline DU11-11 & 211 & 44 & 31 & - & 0.68 & - & DC17-2 & 5898 & 102 & - & - & - & - \\
\hline DU11-12 & 222 & 43 & 31 & - & 0.77 & - & DC18 & 5696 & 254 & 43 & 1.5 & 1.02 & 1.02 \\
\hline DU11-13 & 47 & 25 & 33 & - & 0.40 & - & DC19-1 & 1552 & 212 & 42 & 0.9 & 0.83 & 1.02 \\
\hline DU11-14 & 21 & 25 & 33 & - & 0.18 & - & DC19-2 & 3465 & 230 & 42 & 0.9 & 0.83 & 1.09 \\
\hline DU11-15 & 24 & 25 & 33 & - & 0.21 & - & DC19-3 & 1357 & 86 & 44 & 2.0 & 1.99 & 0.92 \\
\hline DU11-16 & 88 & 25 & 33 & - & 0.75 & - & DC19-4 & 3059 & 86 & 44 & 2.0 & 1.99 & - \\
\hline DU12 & 2870 & 99 & 37 & 0.5 & 0.78 & 0.42 & DC20 & 4520 & 177 & 37 & 0.5 & 0.39 & 1.02 \\
\hline DU13-1 & 729 & 42 & 43 & 1.3 & 1.74 & 1.25 & DC21 & 6808 & 173 & 45 & 0.4 & 0.46 & 1.24 \\
\hline DU13-2 & 334 & 29 & 43 & 1.8 & 1.77 & 1.81 & DC22-1 & 8838 & 311 & 35 & 0.4 & 0.22 & 0.79 \\
\hline DU14-1 & 564 & 49 & 35 & 1.5 & 1.67 & 1.08 & DC22-2 & 7745 & 311 & 35 & 0.4 & 0.22 & 0.69 \\
\hline DU14-2 & 613 & 58 & 35 & 1.3 & 1.23 & 0.94 & DC22-3 & 7935 & 311 & 35 & 0.4 & 0.22 & 0.71 \\
\hline DU14-3 & 522 & 70 & 35 & 1.0 & 0.72 & 0.72 & DC23 & 24400 & 210 & 38 & 0.5 & 0.40 & 1.15 \\
\hline DU14-4 & 598 & 74 & 35 & 0.9 & 0.67 & 0.65 & DC24 & 5600 & 113 & 45 & 0.5 & - & - \\
\hline DU14-5 & 683 & 77 & 35 & 0.8 & 0.64 & 0.58 & DC25 & 9252 & 233 & 35 & 0.4 & 0.21 & 0.86 \\
\hline DU15-1 & 193 & 21 & 33 & 2.3 & 1.32 & 1.54 & DC26 & 1067 & 70 & - & - & - & - \\
\hline DU15-2 & 362 & 27 & 34 & 1.9 & 1.52 & 1.32 & DC27-1 & 2206 & 118 & - & - & - & - \\
\hline DU15-3 & 407 & 28 & 44 & 2.7 & 3.11 & 2.69 & DC27-2 & 2170 & 118 & - & - & - & - \\
\hline DU16 & 974 & 35 & - & - & 2.66 & - & DC28-1 & 676 & 66 & 39 & 0.7 & 0.58 & 1.20 \\
\hline DU17-1 & 2369 & 288 & 45 & 0.3 & 0.24 & 0.27 & DC28-2 & 1210 & 100 & 43 & 0.6 & 0.58 & 0.94 \\
\hline DU17-2 & 2668 & 265 & 45 & 0.3 & 0.20 & 0.27 & DC29 & 124 & 45 & 28 & 0.6 & 0.33 & 1.35 \\
\hline DU18-1 & 92 & 27 & - & - & 0.79 & - & DC30 & 427 & 47 & 37 & 0.9 & 0.70 & 1.18 \\
\hline DU18-2 & 111 & 29 & - & - & 0.81 & - & DC31 & 1370 & 85 & 45 & 0.7 & 0.72 & 0.87 \\
\hline DU18-3 & 113 & 31 & - & - & 0.71 & - & DC32-1 & 9550 & 207 & 33 & 0.5 & 0.31 & 0.86 \\
\hline DU18-4 & 99 & 34 & - & - & 0.53 & - & DC32-2 & 9128 & 207 & 33 & 0.5 & 0.31 & 0.83 \\
\hline DU18-5 & 112 & 27 & - & - & 0.96 & - & DC33 & 7579 & 115 & 41 & 0.6 & 0.46 & 1.07 \\
\hline DU18-6 & 101 & 27 & - & - & 0.86 & - & DC34-1 & 196 & 90 & 30 & 0.5 & 0.30 & 0.67 \\
\hline DU19 & 1950 & 36 & 44 & 5.5 & 6.40 & 5.52 & DC34-2 & 233 & 90 & 30 & 0.5 & 0.30 & 0.69 \\
\hline DU20-1 & 365 & 90 & 30 & 0.5 & 0.37 & 0.31 & DC34-3 & 350 & 90 & 30 & 0.5 & 0.30 & 0.83 \\
\hline DU20-2 & 435 & 90 & 30 & 0.5 & 0.38 & 0.31 & DC35 & 4500 & 135 & - & - & - & - \\
\hline DU20-3 & 431 & 90 & 30 & 0.5 & 0.30 & 0.31 & DC36-1 & 2215 & 65 & - & - & - & - \\
\hline DU21-1 & 82 & 42 & 36 & 1.3 & 0.89 & 0.97 & DC36-2 & 2680 & 94 & - & - & - & - \\
\hline
\end{tabular}

${ }^{\mathrm{a}} \bar{\sigma}_{\mathrm{vm}}=$ mean effective overburden stress along shaft. ${ }^{\mathrm{b}} \bar{\phi}_{\mathrm{tc}}=$ effective stress friction angle $(\bar{\phi})$ for triaxial compression test. ${ }^{\mathrm{c}} K_{o}=\left(1-\sin \bar{\phi}_{\mathrm{tc}}\right) \mathrm{OCR}{ }^{\sin \bar{\phi}_{\mathrm{c}}}$, based on Mayne and Kulhawy [10]. ${ }^{\mathrm{d}} \beta_{\mathrm{m}}$ was calculated using Eq. (10). ${ }^{\mathrm{e}} \beta_{\mathrm{p}}$ was calculated using Eq. (9) and the suggested $\mathrm{K} / \mathrm{K}_{\mathrm{o}}$ values of this study. 
Table 3. Summary of analysis data for undrained loading.

\begin{tabular}{|c|c|c|c|c|c|c|c|c|}
\hline $\begin{array}{c}\text { Site/Case } \\
\text { No. }\end{array}$ & $\begin{array}{c}\mathrm{Q}_{\mathrm{s}} \\
(\mathrm{kN})\end{array}$ & $\begin{array}{c}\bar{\sigma}_{\mathrm{vm}}{ }^{\mathrm{a}} \\
\left(\mathrm{kN} / \mathrm{m}^{2}\right)\end{array}$ & $\begin{array}{c}\mathrm{s}_{\mathrm{u}}(\mathrm{CIUC}) \\
\left(\mathrm{kN} / \mathrm{m}^{2}\right)\end{array}$ & $\mathrm{K}_{\mathrm{o}}^{\mathrm{b}}$ & $\alpha_{\text {CIUC }}$ & $\beta_{\mathrm{m}}^{\mathrm{c}}$ & $\beta_{\mathrm{p}}{ }^{\mathrm{d}}$ & $\lambda$ \\
\hline UU1 & 525 & 37 & 102 & 2.76 & 0.61 & 1.71 & 1.71 & 0.26 \\
\hline UU2-1 & 334 & 19 & 307 & 5.11 & 0.23 & 2.98 & 2.89 & 0.11 \\
\hline UU2-2 & 356 & 19 & 307 & 5.11 & 0.25 & 3.65 & 2.89 & 0.12 \\
\hline UU3-1 & 301 & 25 & 68 & 3.67 & 0.63 & 1.75 & 2.37 & 0.27 \\
\hline UU3-2 & 319 & 25 & 68 & 3.67 & 0.67 & 1.87 & 2.37 & 0.28 \\
\hline UU4 & 290 & 40 & 59 & - & 0.56 & 3.09 & - & 0.21 \\
\hline UU5 & 1177 & 343 & 285 & 1.04 & 0.32 & 0.33 & 0.47 & 0.10 \\
\hline UU6 & 220 & 29 & 100 & 3.10 & 0.35 & 1.22 & 1.85 & 0.15 \\
\hline UU7 & 125 & 23 & 112 & 2.52 & 0.37 & 2.05 & 1.76 & 0.17 \\
\hline UU8 & 270 & 16 & 95 & 4.71 & 0.61 & 3.12 & 3.43 & 0.28 \\
\hline UU9-1 & 402 & 63 & 56 & - & 0.81 & 0.74 & - & 0.26 \\
\hline UU9-2 & 451 & 59 & 57 & - & 0.69 & 0.69 & - & 0.23 \\
\hline UU9-3 & 323 & 40 & 57 & - & 0.66 & 0.95 & - & 0.24 \\
\hline \multirow[t]{2}{*}{ UU10-1 } & 194 & 34 & 26 & 0.61 & 0.87 & 0.67 & 0.40 & 0.26 \\
\hline & 194 & 34 & 32 & 0.61 & 0.71 & - & - & 0.23 \\
\hline \multirow[t]{2}{*}{ UU10-2 } & 182 & 34 & 26 & 0.61 & 0.88 & 0.68 & 0.40 & 0.27 \\
\hline & 182 & 34 & 32 & 0.61 & 0.72 & - & - & 0.23 \\
\hline UU11 & 616 & 80 & 96 & 1.22 & 0.32 & 0.39 & 0.65 & 0.11 \\
\hline UU12-1 & 618 & 43 & 21 & 0.84 & 0.84 & 0.31 & 0.50 & 0.21 \\
\hline \multirow[t]{3}{*}{ UU13-1 } & 480 & 29 & 74 & 2.42 & 0.56 & 1.42 & 1.44 & 0.23 \\
\hline & 480 & 29 & 178 & - & 0.26 & - & - & 0.11 \\
\hline & 480 & 29 & 165 & - & 0.27 & - & - & 0.11 \\
\hline \multirow[t]{3}{*}{ UU13-2 } & 943 & 34 & 86 & 2.32 & 0.63 & 1.59 & 1.38 & 0.26 \\
\hline & 943 & 34 & 180 & - & 0.30 & - & - & 0.14 \\
\hline & 943 & 34 & 166 & - & 0.33 & - & - & 0.15 \\
\hline \multirow[t]{3}{*}{ UU13-3 } & 147 & 26 & 68 & 2.55 & 0.62 & 1.62 & 1.51 & 0.26 \\
\hline & 147 & 26 & 179 & - & 0.25 & - & - & 0.11 \\
\hline & 147 & 26 & 165 & - & 0.26 & - & - & 0.12 \\
\hline UU14-1 & 2044 & 57 & 58 & 0.90 & 0.64 & 0.65 & 0.68 & 0.21 \\
\hline UU14-2 & 2044 & 57 & 45 & - & 0.82 & - & - & 0.25 \\
\hline UU14-3 & 2044 & 57 & 53 & - & 0.70 & - & - & 0.23 \\
\hline UU15-1 & 448 & 29 & 182 & 1.80 & 0.40 & 2.48 & 1.41 & 0.18 \\
\hline UU15-2 & 723 & 44 & 182 & 1.41 & 0.42 & 1.73 & 1.11 & 0.19 \\
\hline UU16 & 8896 & 296 & 120 & 0.53 & 0.56 & 0.23 & 0.24 & 0.13 \\
\hline UU17 & 6316 & 285 & 120 & 0.50 & 0.40 & 0.19 & 0.25 & 0.09 \\
\hline UU18 & 11565 & 142 & 100 & 0.88 & 0.51 & 0.36 & 0.40 & 0.15 \\
\hline UU19 & 3558 & 75 & 90 & 0.88 & 0.40 & 0.47 & 0.38 & 0.14 \\
\hline UU20-1 & 614 & 51 & 94 & 1.01 & 0.42 & 0.77 & 0.60 & 0.16 \\
\hline UU20-2 & 667 & 51 & 94 & 1.01 & 0.45 & 0.83 & 0.60 & 0.18 \\
\hline
\end{tabular}

\begin{tabular}{|c|c|c|c|c|c|c|c|c|}
\hline $\begin{array}{c}\text { Site/Case } \\
\text { No. }\end{array}$ & $\begin{array}{c}\mathrm{Q}_{\mathrm{s}} \\
(\mathrm{kN})\end{array}$ & $\begin{array}{c}\bar{\sigma}_{\mathrm{vm}}{ }^{\mathrm{a}} \\
\left(\mathrm{kN} / \mathrm{m}^{2}\right)\end{array}$ & $\begin{array}{c}\mathrm{s}_{\mathrm{u}}(\mathrm{CIUC}) \\
\left(\mathrm{kN} / \mathrm{m}^{2}\right)\end{array}$ & $\mathrm{K}_{\mathrm{o}}^{\mathrm{b}}$ & $\alpha_{\text {CIUC }}$ & $\beta_{\mathrm{m}}{ }^{\mathrm{c}}$ & $\beta_{\mathrm{p}}{ }^{\mathrm{d}}$ & $\lambda$ \\
\hline $\mathrm{UC} 1$ & 395 & 55 & 147 & 1.19 & 0.34 & 0.92 & 0.74 & 0.14 \\
\hline UC2 & 1516 & 54 & 90 & 0.93 & 0.56 & 0.95 & 0.53 & 0.22 \\
\hline UC3 & 8162 & 148 & 100 & 0.41 & 0.50 & 0.34 & 0.35 & 0.14 \\
\hline UC4 & 19706 & 236 & 120 & 0.50 & 0.50 & 0.25 & 0.25 & 0.13 \\
\hline UC5 & 13406 & 296 & 120 & 0.53 & 0.52 & 0.21 & 0.24 & 0.12 \\
\hline UC6 & 9528 & 197 & 120 & 0.89 & 0.45 & 0.31 & 0.38 & 0.12 \\
\hline UC7 & 11521 & 231 & 120 & 0.49 & 0.40 & 0.21 & 0.26 & 0.10 \\
\hline UC8 & 4448 & 90 & 100 & 0.79 & 0.40 & 0.45 & 0.37 & 0.14 \\
\hline UC9 & 290 & 39 & 24 & 0.74 & 0.88 & 0.54 & 0.44 & 0.11 \\
\hline \multirow{2}{*}{ UC10-1 } & 178 & 30 & 132 & 2.14 & 0.27 & 1.19 & 1.38 & 0.10 \\
\hline & 178 & 30 & 137 & - & 0.26 & - & - & 0.10 \\
\hline \multirow[t]{2}{*}{ UC10-2 } & 383 & 45 & 118 & 1.74 & 0.35 & 0.91 & 1.13 & 0.13 \\
\hline & 383 & 45 & 109 & & 0.38 & - & - & 0.14 \\
\hline \multirow[t]{2}{*}{ UC10-2 } & 890 & 67 & 136 & 1.45 & 0.45 & 0.91 & 1.01 & 0.17 \\
\hline & 890 & 67 & 123 & - & 0.50 & - & - & 0.18 \\
\hline UC11-1 & 636 & 107 & 291 & 1.59 & 0.32 & 0.86 & 1.05 & 0.03 \\
\hline UC11-2 & 2500 & 119 & 303 & 1.40 & 0.41 & 1.04 & 0.93 & 0.10 \\
\hline UC11-3 & 2400 & 110 & 297 & 1.39 & 0.27 & 0.74 & 0.92 & 0.07 \\
\hline UC11-4 & 1180 & 105 & 340 & 1.40 & 0.29 & 0.89 & 0.93 & 0.04 \\
\hline UC11-5 & 1400 & 97 & 302 & 1.42 & 0.23 & 0.71 & 0.94 & 0.04 \\
\hline \multirow[t]{2}{*}{ UC12-1 } & 946 & 70 & 119 & 1.37 & 0.40 & 0.69 & 0.92 & 0.18 \\
\hline & 946 & 70 & 94 & - & 0.51 & - & - & 0.22 \\
\hline \multirow[t]{2}{*}{ UC12-2 } & 1067 & 70 & 119 & 1.37 & 0.54 & 0.91 & 0.92 & 0.21 \\
\hline & 1067 & 70 & 94 & - & 0.68 & - & - & 0.25 \\
\hline \multirow[t]{2}{*}{ UC12-3 } & 2130 & 105 & 137 & 1.27 & 0.37 & 0.48 & 0.63 & 0.18 \\
\hline & 2130 & 105 & 120 & - & 0.42 & - & - & 0.20 \\
\hline UC13-1 & 41 & 28 & 48 & 2.00 & 0.63 & 1.07 & 1.29 & 0.24 \\
\hline UC13-2 & 74 & 36 & 50 & 1.85 & 0.71 & 0.99 & 1.15 & 0.26 \\
\hline UC13-3 & 122 & 40 & 57 & 1.83 & 0.80 & 1.15 & 1.09 & 0.30 \\
\hline UC13-4 & 129 & 41 & 68 & 1.83 & 0.68 & 1.14 & 1.09 & 0.26 \\
\hline \multirow[t]{3}{*}{ UC14-1 } & 2355 & 83 & 192 & 1.56 & 0.45 & 1.04 & 1.32 & 0.18 \\
\hline & 2355 & 83 & 208 & - & 0.41 & - & - & 0.17 \\
\hline & 2355 & 83 & 193 & - & 0.45 & - & - & 0.18 \\
\hline \multirow[t]{3}{*}{ UC14-2 } & 2028 & 83 & 192 & 1.56 & 0.37 & 0.86 & 1.32 & 0.15 \\
\hline & 2028 & 83 & 208 & - & 0.34 & - & - & 0.14 \\
\hline & 2028 & 83 & 193 & - & 0.37 & - & - & 0.15 \\
\hline \multirow[t]{2}{*}{ UC15-1 } & 329 & 60 & 140 & 1.11 & 0.31 & 0.70 & 0.72 & 0.12 \\
\hline & 329 & 60 & 186 & - & 0.25 & - & - & 0.10 \\
\hline UC15-2 & 3053 & 160 & 260 & 0.99 & 0.40 & 0.71 & 0.67 & 0.17 \\
\hline
\end{tabular}

${ }^{\mathrm{a}} \bar{\sigma}_{\mathrm{vm}}=$ mean effective overburden stress along shaft. ${ }^{\mathrm{b}} K_{o}=\left(1-\sin \bar{\phi}_{\mathrm{tc}}\right) \mathrm{OCR}{ }^{\sin \bar{\phi}_{\mathrm{tc}}}$ , based on Mayne and Kulhawy [10]. ${ }^{\mathrm{c}} \beta_{\mathrm{m}}$ was calculated using Eq. (10). ${ }^{\mathrm{d}} \beta_{\mathrm{p}}$ was calculated using Eq. (9) and the suggested $\mathrm{K} / \mathrm{K}_{\mathrm{o}}$ values of this study.

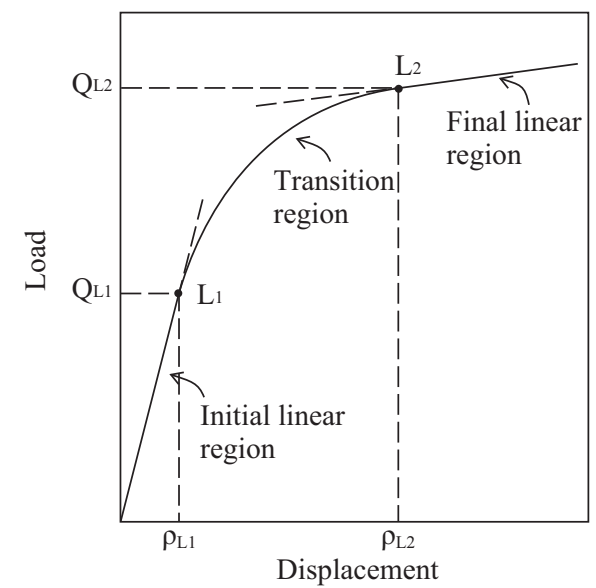

Fig. 2. Regions of load-displacement curve. loading. They also developed the relationships between $\mathrm{L}_{2}$ and other interpretation criteria from lower bound to higher bound. Therefore, these interrelationships among interpretation criteria are also used to infer the required $\mathrm{L}_{2}$ if the load test data are insufficient or are terminated prematurely.

Table 4 lists the reference sources and soil strength test types of all load test case histories in Tables 2 and 3. These load tests were conducted on various types of soil around the world and at different times. For convenience, Table 5 lists the ranges of foundation geometry and the interpreted capacities from the database, along with the data standard deviation (SD) and the coefficient of variation (COV), which is the standard deviation divided by the mean. As can be seen, the ranges are broad, but the results for drained and undrained analyses are roughly comparable. 
Table 4. Reference sources of shaft load tests in Tables 2 and 3.

\begin{tabular}{|c|c|c|c|}
\hline \multirow{2}{*}{ Site No. } & \multicolumn{2}{|c|}{ Test type } & \multirow{2}{*}{ Reference sources } \\
\hline & $\mathrm{S}_{\mathrm{u}}{ }^{\mathrm{a}}$ & $\bar{\phi}^{\mathrm{b}}$ & \\
\hline DU1, 15; UU2, 3 & $\mathrm{UC}$ & SPT-N & $\begin{array}{l}\text { Harza Engineering Co. (1978)“Foundation uplift tests - Missouri Basin Power Project East Transmission”, } \\
\text { for Basin Electric Power Coop., Bismarck, } 26 \text { p. }\end{array}$ \\
\hline DU2 & - & SPT-N & $\begin{array}{l}\text { Ohio Edison Co. (early 1960s) "Test program proves feasibility of concrete cylinder anchors for steel trans- } \\
\text { mission towers for Ohio Edison Co.", Akron, } 8 \text { p. }\end{array}$ \\
\hline DU3 & - & SPT-N & $\begin{array}{l}\text { Virginia Electric and Power Co. (1967) "Tests on piles and caissons, slurry-Hopewell } 230 \mathrm{KV} \text {; Bacons Castle, } \\
\text { Virginia", } 15 \text { p. }\end{array}$ \\
\hline DU4; DC1 & - & SPT-N & $\begin{array}{l}\text { Florida Testing Laboratories (1965) “Combined section test pile, tension and compression”, for Florida Power } \\
\text { Corp., St. Petersburg, } 9 \text { p. }\end{array}$ \\
\hline DU5 & - & SPT-N & $\begin{array}{l}\text { Florida Testing Laboratories (1966) "Piles research for design parameters", for Florida Power Corp., St. } \\
\text { Petersburg, } 69 \text { p. }\end{array}$ \\
\hline DU6; DC2 & - & SPT-N & $\begin{array}{l}\text { Ismael, NF and Klym, TW (1979) "Uplift and bearing capacity of short piers in sand", J. Geotechnical Eng. } \\
\text { Div., ASCE, 105(GT5), 579-594. }\end{array}$ \\
\hline DU7 & - & SPT-N & $\begin{array}{l}\text { Stern, LI, Bose, SK and King, RD (1976) "Uplift capacity of poured-in-place cylindrical caissons", Paper A } \\
76 \text { 153-9, IEEE PES Winter Meeting, New York, } 9 \text { p. }\end{array}$ \\
\hline DU8; UU11 & UC & SPT-N & $\begin{array}{l}\text { Sowa, VA (1970) "Pulling capacity of concrete cast-in-situ bored piles", Canadian Geotechnical J., 7(4), } \\
482-493 \text {. }\end{array}$ \\
\hline DU9 & - & SPT-N & $\begin{array}{l}\text { Stone and Webster Engineering Corp. (1969) "Report on pull test on auger piles, Branchburg to Ramapo } \\
\text { Line", Public Service Electric and Gas Co., Newark, } 18 \text { p. }\end{array}$ \\
\hline DU10; UU15 & CIUC & CPT & $\begin{array}{l}\text { Briaud, J-L, Pacal, AJ and Shively, AW (1984) "Power line foundation design using the pressuremeter", Proc., } \\
1^{\text {st }} \text { Intl. Conf. on Case Histories in Geotechnical Eng. (1), Rolla, 279-283. }\end{array}$ \\
\hline DU11; UU9 & DSS & CPT & $\begin{array}{l}\text { Tucker, KD (1987) "Uplift capacity of drilled shafts and driven piles in granular materials", Foundations for } \\
\text { Transmission Line Towers (GSP 8), Ed. J-L Briaud, ASCE, New York, 142-159. }\end{array}$ \\
\hline DU12; DC3 & - & SPT-N & $\begin{array}{l}\text { HDR Infrastructure (1986) "Preliminary foundation report and summary of load test program", I-275, How- } \\
\text { ard Frankling Bridge, Tampa, } 77 \text { p. }\end{array}$ \\
\hline DU13 & - & CPT & $\begin{array}{l}\text { Konstantinidis, B, Pacal, AJ and Shively, AW (1987) "Uplift capacity of drilled piers in desert soils: a case } \\
\text { history", Foundations for Transmission Line Towers (GSP 8), Ed. J-L Briaud, ASCE, New York, 128-141. }\end{array}$ \\
\hline DU14 & - & SPT-N & $\begin{array}{l}\text { Marsico, R, Retallack, RL and Tedesco, PA, (1976) "Report on pile testing for AEP transmission lines - Part I } \\
\text { and II", Transactions on Power Apparatus and Systems, IEEE, 95(6), 1795-1813. }\end{array}$ \\
\hline DU16 & - & SPT-N & Pacific Power and Light Co. (1980) "Pier uplift test”, Portland, 20 p. \\
\hline DU17 & - & SPT-N & Sacre, AS (1977) "Study of pullout resistance of drilled shafts”, MS Thesis, Univ. of Texas, Austin, 164 p. \\
\hline DU18; UU7, 8 & UU & - & $\begin{array}{l}\text { Paterson, G and Urie, RL (1964) "Uplift resistance tests on full sizes transmission tower foundations", Proc., } \\
\text { CIGRE (1), Paper 203, Paris, } 19 \text { p. }\end{array}$ \\
\hline DU19 & - & SPT-N & $\begin{array}{l}\text { Woodward-Clyde Consultants (1980) "Geotechnical investigation for the Miguel-Imperial valley } 500 \mathrm{KV} \\
\text { transmission line (Tower sites } 25 \text { through 213)", for San Diego Gas and Electric Co., San Diego, } 62 \mathrm{p} \text {. }\end{array}$ \\
\hline DU20; DC34 & - & CPT & $\begin{array}{l}\text { Chambon, P., Corté J-F (1991) Étude sur modèles réduits centrifugés. Application aux tunnels à faible } \\
\text { profondeur en terrain meuble pulvérulent, Études et Recherches des Laboratoires des Ponts et Chaussées, } \\
\text { série Géotechnique, GT 48, } 163 \text {. }\end{array}$ \\
\hline & & & $\begin{array}{l}\text { Neves, M, Mestat, P, Frank, R and Degny, E (2001) "Research on the behavior of bored piles - I. in situ and } \\
\text { laboratory experiments", CESAR-LCPC, 231, 39-54. }\end{array}$ \\
\hline DU21 & - & CPT & $\begin{array}{l}\text { Sven, K, Johan, C and Lars, D (2006) "Tension test on bored piles in sand", Symp. Intl. ELU/ULS, Paris, } \\
\text { 87-94. }\end{array}$ \\
\hline DC4 & - & SPT-N & $\begin{array}{l}\text { Jain, GS and Gupta, SP (1968) “Comparative study of multi-underreamed pile with large diameter pile in } \\
\text { sandy soil”, Proc., } 3^{\text {rd }} \text { Budapest Conf. on Soil Mechanics and Foundation Eng., Budapest, 563-570. }\end{array}$ \\
\hline DC5 & - & SPT-N & $\begin{array}{l}\text { O’Neill, MW and Reese, LC (1978) "Load transfer in a slender drilled pier in sand", Preprint } 3141 \text {, ASCE } \\
\text { Spring Convention, Pittsburgh, } 30 \text { p. }\end{array}$ \\
\hline DC6, 7 & - & SPT-N & $\begin{array}{l}\text { Reese, LG and O’Neill, MW (1988) "Field load tests of drilled shafts", Proc., 1st Intl. Geotechnical Seminar } \\
\text { on Deep Foundations on Bored and Auger Piles, }\end{array}$ \\
\hline DC8 & - & CPT & $\begin{array}{l}\text { Brusey, WG and York, DL (1991) "Pile test program at John F. Kennedy International Airport", Foundations } \\
\text { Profondes, Ecole Nationale des Ponts et Chausses, Paris, 379-387. }\end{array}$ \\
\hline DC9 & - & $\mathrm{CPT}$ & $\begin{array}{l}\text { Stuckrath, L and Descoeudres, F (1991) "Large-scale pile tests in an instrumented test pit", Foundations } \\
\text { Profondes, Ecole Nationale des Ponts et Chausses, Paris, 301-308. }\end{array}$ \\
\hline DC10 & - & CPT & $\begin{array}{l}\text { Roscoe, GH (1983) "Behavior of flight auger bored piles in sand", Proc., Intl. Conf. on Advances in Piling and } \\
\text { Ground Treatment for Foundations, ICE, London, 241-250. }\end{array}$ \\
\hline DC11 & - & SPT-N & $\begin{array}{l}\text { Azevedo, N, Jr. (1991) "Load transfer in bored pile in residual soil", Proc., } 9^{\text {th }} \text { Pan-American Conf. on Soil } \\
\text { Mechanics and Foundation Eng. (2), 569-576. }\end{array}$ \\
\hline DC12 & - & SPT-N & $\begin{array}{l}\text { Farr, S, and Aurora, RP (1981) "Behavior of instrumented pier in gravelly sand", Drilled Piers and Caissons, } \\
\text { Ed. MW, O’Neill, ASCE, New York, 53-65. }\end{array}$ \\
\hline DC13 & - & - & $\begin{array}{l}\text { Reese, LC (1982) “Analysis of instrumented drilled shafts subjected to axial load, Sunshine Skyway Bridge”, } \\
\text { Report GR81-20, Geotechnical Eng. Center, Univ. of Texas, Austin, } 69 \text { p. }\end{array}$ \\
\hline DC14 & - & CPT & $\begin{array}{l}\text { Fujita, K (1986) "Pile construction and related problems", Proc., Intl. Conf. on Deep Foundations (2), Beijing, } \\
\text { 137-155. }\end{array}$ \\
\hline
\end{tabular}


Table 4. (Continued)

\begin{tabular}{|c|c|c|c|}
\hline \multirow{2}{*}{ Site No. } & \multicolumn{2}{|c|}{ Test type } & \multirow{2}{*}{ Reference sources } \\
\hline & $\mathrm{s}_{\mathrm{u}}{ }^{\mathrm{a}}$ & $\bar{\phi}^{\mathrm{b}}$ & \\
\hline $\mathrm{DC} 15$ & - & $\mathrm{CPT}$ & $\begin{array}{l}\text { Long, JH and Reese, LC (1983) "Analysis of field tests of drilled shafts", Report to Farr Foundation Co., } \\
\text { Univ. of Texas, Austin, } 34 \text { p. }\end{array}$ \\
\hline DC16 & - & - & $\begin{array}{l}\text { Cernak, B (1976) "Time effect of suspension on behavior of piers", Proc., } 6^{\text {th }} \text { European Conf. on Soil Me- } \\
\text { chanics and Foundation Eng. (1.1), Vienna, 111-114. }\end{array}$ \\
\hline DC17 & - & - & $\begin{array}{l}\text { Schmertmann and Crapps, Inc. (1985) "I-595 Viaduct Load Test Program”, Job 86095-3406, for Florida Dept. } \\
\text { of Transportation, Gainesville, } 54 \text { p. }\end{array}$ \\
\hline DC18 & - & SPT-N & $\begin{array}{l}\text { Baker, CN, Jr. (1991) "Prediction and performance of drilled shafts constructed under slurry", } 16^{\text {th }} \text { Annual } \\
\text { Deep Foundations Institute Conf., Chicago, 155-172. }\end{array}$ \\
\hline DC19 & - & SPT-N & $\begin{array}{l}\text { Franke, E and Garbrecht, D (1977) "Test loading on } 8 \text { large bored piles in sand", Proc., } 9^{\text {th }} \text { Intl. Conf. on Soil } \\
\text { Mechanics and Foundation Eng. (1), Tokyo, 529-532. }\end{array}$ \\
\hline DC20 & - & $\mathrm{CPT}$ & $\begin{array}{l}\text { Caputo, V and Viggiani, C (1988) "Some experiences with bored and auger piles in Naples area", Proc., } 1^{\text {st }} \\
\text { Intl. Geotechnical Seminar on Deep Foundations on Bored and Auger Piles, Ghent, 273-281. }\end{array}$ \\
\hline DC21 & - & SPT-N & $\begin{array}{l}\text { Frank, R, Kalteziotis, N, Bustamante, M, Christoulas, S and Zervogiannis, H (1991) "Evaluation of per- } \\
\text { formance of two piles using pressuremeter method", J. Geotechnical Eng., ASCE, 117(5), 695-713. }\end{array}$ \\
\hline DC22 & - & SPT-N & $\begin{array}{l}\text { Chang, MF and Lo, SS (1990) "Behavior of some axially loaded cast-in-place piles", Proc., } 10^{\text {th }} \text { Southeast } \\
\text { Asian Geotechnical Conf. (1), Taipei, 327-332. }\end{array}$ \\
\hline DC23 & - & SPT-N & $\begin{array}{l}\text { Hirayama, H (1990) "Load-settlement analysis for bored piles using hyperbolic transfer functions", Soils and } \\
\text { Foundations, 30(1), 55-64. }\end{array}$ \\
\hline DC24 & - & $\mathrm{CPT}$ & $\begin{array}{l}\text { Brandl, H (1985) "Bearing capacity of piers and piles with large diameters", Proc., } 11^{\text {th }} \text { Intl. Conf. on Soil } \\
\text { Mechanics and Foundation Eng. (3), San Francisco, 1525-1530. }\end{array}$ \\
\hline DC25 & - & CPT & $\begin{array}{l}\text { Wang, YZ (1986) "Design and construction of large bored piles over Yellow River", Proc., Intl. Conf. on Deep } \\
\text { Foundations (2), Beijing, 124-129. }\end{array}$ \\
\hline DC26 & - & CPT & $\begin{array}{l}\text { Martins, FF and Martins, JB (1989) "CPT and pile tests in granitic residual soils", Proc., } 12^{\text {th }} \text { Intl. Conf. on } \\
\text { Soil Mechanics and Foundation Eng. (1), Rio de Janeiro, 529-531. }\end{array}$ \\
\hline $\mathrm{DC} 27$ & - & - & $\begin{array}{l}\text { Maertens, J (1985) "Comparative tests on bored and driven piles at Kallo", Belgian Geotechnical Volume, } \\
\text { Published for the } 1985 \text { Golden Jubilee, ISSMFE, 31-38. }\end{array}$ \\
\hline DC28, 29, 30, 31 & - & $\mathrm{CPT}$ & $\begin{array}{l}\text { Burch, SB, Parra, F, Townsend, FC and McVay, MC (1988) "Design guidelines for drilled shaft foundations", } \\
\text { Univ. of Florida, Gainesville, } 141 \text { p. }\end{array}$ \\
\hline DC32 & - & SPT-N & $\begin{array}{l}\text { Diagnostic Engineering Consultants, Ltd. (2001). "Report on compression load test of bored piles for Taiwan } \\
\text { high speed rail project", Taiwan. }\end{array}$ \\
\hline DC33 & - & SPT-N & $\begin{array}{l}\text { Hai Tain Engeering Ltd. (2002). "Report on compression load test of bored piles at Daja township", } \\
\text { Tai-Chung County, Taiwan. }\end{array}$ \\
\hline DC 35,36 & - & - & $\begin{array}{l}\text { Thasnanipan, N, Maung, AW, Ganeshan, B and Teparaksa W (1998). "Design, construction and behavior of } \\
\text { bored cast-in-situ concrete piles in Bangkok subsoils", Proc., 4th Intl. Conf. on Case Histories in Geotechnical } \\
\text { Eng., Louis Missouri, 281-287. }\end{array}$ \\
\hline UU1 & $\mathrm{UC}$ & $\mathrm{TC}$ & $\begin{array}{l}\text { Mckenzie, RJ (1971). "Uplift testing of prototype transmission tower footings", Proc., } 1^{\text {st }} \text { Australia-New } \\
\text { Zealand Conf. on Geomechanics (1), Melbourne, 283-290. }\end{array}$ \\
\hline UU4 & UC & $\mathrm{TC}$ & $\begin{array}{l}\text { Los Angeles Department of Water and Power (1967) "Footing tests for Pacific Intertie D-C system trans- } \\
\text { mission line", Los Angeles, } 37 \text {. }\end{array}$ \\
\hline UU5 & UU & $\mathrm{TC}$ & $\begin{array}{l}\text { Pearce, RA and Brassow, CL (1979). "Pull-out load test of a drilled pier in very stiff Beaumont clay", Symp. } \\
\text { on Deep Foundation, Ed. FM Fuller, ASCE, New York, 331-342. }\end{array}$ \\
\hline UU6 & UU & SPT-N & $\begin{array}{l}\text { Wichita Testing Co. (1977). "Final report - steel Tower foundation test", for Kansas Gas and Electric Co., } \\
\text { Wichita, } 36 .\end{array}$ \\
\hline UU10 & UU, UC & CPT & $\begin{array}{l}\text { Tang, NC, Shen, HR and Liu, SG (1983). "Static uplift capacity of bored piles", Proc., Intl. Conf. on Advances } \\
\text { in Piling and Ground Treatment, ICE, London, 197-202. }\end{array}$ \\
\hline UU12; UC9 & CIUC & $\mathrm{TC}$ & $\begin{array}{l}\text { Radhakrishna, HS, Cragg, CBH, Tsang, R and Bozozuk, M (1986), "Uplift and compression behavior of } \\
\text { drilled piers in Leda clay", Proc., } 39^{\text {th }} \text { Canadian Geotechnical Conf., Ottawa, 123-130. }\end{array}$ \\
\hline UU13 & $\mathrm{UU}, \mathrm{UC}$ & $\mathrm{TC}$ & $\begin{array}{l}\text { Adams, JI and Radhakrishna, HS (1970). "Uplift resistance of augered footings in fissured clay", Ontario } \\
\text { Hydro Research Quarterly, 22(1), 10-16. }\end{array}$ \\
\hline UU14 & $\begin{array}{l}\text { CIUC } \\
\mathrm{UU}, \mathrm{UC}\end{array}$ & $\mathrm{TC}$ & $\begin{array}{l}\text { Ismael, NF and Klym, TW (1978). "Behavior of rigid piers in layered cohesive soils", J. Geotechnical Eng. } \\
\text { Div., ASCE, 104(GT8), 1061-1074. }\end{array}$ \\
\hline UU16; UC5 & UU, UC & SPT-N & $\begin{array}{l}\text { Diagnostic Engineering Consultants, Ltd. (2002). "Report on uplift and compression load test of bored piles } \\
\text { for Makoto bank", Taiwan, Taipei. }\end{array}$ \\
\hline UU17;UC6 & UU & SPT-N & $\begin{array}{l}\text { Fubon Bank Corp. (1996). "Report on uplift and compression load test of bored piles for Fubon bank", } \\
\text { Taiwan, Taipei. }\end{array}$ \\
\hline UU18 & UU & SPT-N & $\begin{array}{l}\text { Diagnostic Engineering Consultants, Ltd. (2002). "Report on uplift load test of bored piles for Hung-Sheng } \\
\text { Debao", Taiwan, Taipei. }\end{array}$ \\
\hline UU19; UC8 & $\mathrm{UC}, \mathrm{UU}$ & SPT-N & $\begin{array}{l}\text { Diagnostic Engineering Consultants, Ltd. (2002). "Report on uplift load test of bored piles for Shin Kong } \\
\text { Insurance Co.", Taiwan, Taipei. }\end{array}$ \\
\hline UU20 & $\mathrm{UU}$ & $\mathrm{TC}$ & $\begin{array}{l}\text { Yajima, J, Aoki, Y, and Shibasaki, F (1993). "Uplift capacity of piles under cyclic load", Proc., 11th Southeast } \\
\text { Asian Geotechnical Conf., Singapore, 601-604. }\end{array}$ \\
\hline
\end{tabular}


Table 4. (Continued)

\begin{tabular}{|c|c|c|c|}
\hline \multirow{2}{*}{ Site No. } & \multicolumn{2}{|c|}{ Test type } & \multirow{2}{*}{ Reference sources } \\
\hline & $\mathrm{S}_{\mathrm{u}}{ }^{\mathrm{a}}$ & $\bar{\phi}^{\mathrm{b}}$ & \\
\hline UU21 & UC & SPT-N & $\begin{array}{l}\text { Reference not available for publication. Summary is given as Case No. } 25 \text { (1983). "Transmission line structure } \\
\text { foundation for uplift-compression loading: load test summaries", Report EL-3160, EPRI, Palo Alto, } 731 \mathrm{p} \text {. }\end{array}$ \\
\hline $\mathrm{UC1}$ & UU, UC & SPT-N & $\begin{array}{l}\text { Diagnostic Engineering Consultants, Ltd. (2002). "Report on compression load test of bored piles for Taipei } \\
\text { Area", Taipei, Taiwan. }\end{array}$ \\
\hline UC2 & UU & SPT-N & $\begin{array}{l}\text { Taiwan Keelung District Court (1995). "Report of compression load test of bored piles for new court build- } \\
\text { ing", Keelung, Taiwan. }\end{array}$ \\
\hline UC3 & UU & SPT-N & $\begin{array}{l}\text { Diagnostic Engineering Consultants, Ltd. (1997). "Report on compression load test of bored piles for Yungho } \\
\text { Sanshing new construction", Taipei, Taiwan. }\end{array}$ \\
\hline UC4 & UU & SPT-N & $\begin{array}{l}\text { Diagnostic Engineering Consultants, Ltd. (2000). "Report on compression load test of bored piles for Taipei } \\
\text { Ta-Chi Bridge", Taipei, Taiwan. }\end{array}$ \\
\hline UC7 & UC & SPT-N & $\begin{array}{l}\text { Tzuchi Culture Center (2002). "Report on compression load test of bored piles for building of Tzuchi Culture } \\
\text { Center", Taipei, Taiwan. }\end{array}$ \\
\hline UC10 & DSS, VST & $\mathrm{TC}$ & $\begin{array}{l}\text { Watt, WG, Kurfurst, PJ and Zeman, ZP (1969). "Comparison of pile load test - skin friction values and } \\
\text { laboratory strength tests", Canadian Geotechnical J., 6(3), 339-352. }\end{array}$ \\
\hline UC11 & UC & $\mathrm{TC}$ & $\begin{array}{l}\text { Jelinek, R, Koreck, HW and Stocker, M (1977). "Load test on } 5 \text { large diameter bored piles in clay", Proc., } 9^{\text {th }} \\
\text { Intl. Conf. on Soil Mechanics and Foundation Eng. (1), } \\
\text { Tokyo, 571-576. }\end{array}$ \\
\hline $\mathrm{UC} 12$ & UU,UC & $\mathrm{TC}$ & $\begin{array}{l}\text { O’Neill, MW and Reese, LC (1970). "Behavior of axially loaded drilled shafts in Beaumont clay", Research } \\
\text { Report 89-8, Univ. of Texas, Austin, } 775 . \\
\text { O'Neill, MW and Reese, LC (1972). "Behavior of bored piles in Beaumont clay", J. Soil Mechanics and } \\
\text { Foundations Div., ASCE, 98(SM2), 195-213. }\end{array}$ \\
\hline UC13 & UC & $\mathrm{TC}$ & DuBose, LA (1955), “Load studies on drilled shaft”, Proc., Highway Research Board (34), 152-162. \\
\hline UC14 & $\begin{array}{l}\text { CIUC } \\
\text { UU, UC }\end{array}$ & TC & $\begin{array}{l}\text { Holtz, RD and Baker, CN (1972). "Some load transfer data on caissons in hard Chicago clay", Proc., ASCE } \\
\text { Conf. on Performance of Earth and Earth Supported Structures (1), Purdue Univ., Lafayette, 1223-1242. }\end{array}$ \\
\hline UC15 & $\mathrm{UU}, \mathrm{UC}$ & $\mathrm{TC}$ & $\begin{array}{l}\text { Bhanot, KL (1968). "Behavior of scaled and full-length cast-in-place concrete piles", Ph.D. Thesis, Univ. of } \\
\text { Alberta, Edmonton, } 336 .\end{array}$ \\
\hline
\end{tabular}

Table 5. Range of geometry and test number of drilled shafts for analysis.

\begin{tabular}{|c|c|c|c|c|c|c|}
\hline \multirow{2}{*}{ Soil type ${ }^{a}$} & \multirow{2}{*}{ No. tests } & & \multicolumn{2}{|c|}{ Shaft geometry (m) } & \multirow{2}{*}{$\mathrm{D} / \mathrm{B}$} & \multirow{2}{*}{ Interpreted $\mathrm{Q}_{\mathrm{s}}^{\mathrm{b}}(\mathrm{kN})$} \\
\hline & & & Depth, D & Dia., B & & \\
\hline \multirow{4}{*}{$\mathrm{D}$} & \multirow{4}{*}{74} & Range & $1.4-62.0$ & $0.14-2.0$ & $2.5-70.5$ & $21-24400$ \\
\hline & & Mean & 11.5 & 0.73 & 16.6 & 2061 \\
\hline & & $\mathrm{SD}$ & 11.3 & 0.36 & 13.4 & 3254 \\
\hline & & $\mathrm{COV}$ & 0.99 & 0.49 & 0.80 & 1.58 \\
\hline \multirow{4}{*}{$\mathrm{U}$} & \multirow{4}{*}{148} & Range & $1.6-77.0$ & $0.18-1.8$ & $1.6-64.2$ & $41-21503$ \\
\hline & & Mean & 14.9 & 0.82 & 17.3 & 2702 \\
\hline & & $\mathrm{SD}$ & 16.6 & 0.38 & 13.4 & 3983 \\
\hline & & $\mathrm{COV}$ & 1.11 & 0.46 & 0.78 & 1.47 \\
\hline
\end{tabular}

${ }^{\mathrm{a}} \mathrm{D}=$ drained; $\mathrm{U}=$ undrained. ${ }^{\mathrm{b}} \mathrm{Q}_{\mathrm{s}}$ was interpreted from $\mathrm{L}_{1}-\mathrm{L}_{2}$ method.

\section{EVALUATION OF SIDE RESISTANCE}

\section{1. $\boldsymbol{\alpha}$ Method}

\section{1) $\alpha-s_{u}$ Correlations}

The value of $\alpha$ can be back-calculated from the field load test results using Eq. (1) and is simplified as follows:

$$
\alpha=\mathrm{Q}_{\mathrm{s}}\left(\mathrm{L}_{2}\right) /\left[\pi \mathrm{B}^{\mathrm{D}} \mathrm{s}_{\mathrm{u}}\right]
$$

where $\mathrm{Q}_{\mathrm{s}}\left(\mathrm{L}_{2}\right)$ = interpreted side resistance using the $\mathrm{L}_{2}$ method, $\mathrm{s}_{\mathrm{u}}=$ mean undrained shear strength over shaft depth (D), and $\mathrm{B}=$ shaft diameter. To standardize the $\alpha-\mathrm{s}_{\mathrm{u}}$ relationship, a unique test type of undrained shear strength from consolidated-isotropically undrained triaxial compression (CIUC) 
Table 6. $\alpha-s_{u}$ correlations for different test and loading types.

\begin{tabular}{|c|c|c|c|c|c|}
\hline \multirow{2}{*}{$\mathrm{s}_{\mathrm{u}}$ test type } & \multirow{2}{*}{ Loading type } & \multirow{2}{*}{ Regression equation } & \multicolumn{3}{|c|}{ Statistics } \\
\hline & & & $\mathrm{n}$ & $r^{2}$ & $\mathrm{SD}$ \\
\hline \multirow{3}{*}{ CIUC } & Compression & $\alpha_{\text {CIUC }}=0.29+0.19 /\left[\mathrm{s}_{\mathrm{u}}(\mathrm{CIUC}) / \mathrm{p}_{\mathrm{a}}\right]$ & 104 & 0.61 & 0.08 \\
\hline & Uplift & $\alpha_{\text {CIUC }}=0.32+0.16 /\left[\mathrm{s}_{\mathrm{u}}(\mathrm{CIUC}) / \mathrm{p}_{\mathrm{a}}\right]$ & 44 & 0.65 & 0.12 \\
\hline & All data & $\alpha_{\text {CIUC }}=0.30+0.17 /\left[\mathrm{s}_{\mathrm{u}}(\mathrm{CIUC}) / \mathrm{p}_{\mathrm{a}}\right]$ & 148 & 0.66 & 0.09 \\
\hline \multirow{3}{*}{ UU } & Compression & $\alpha_{\mathrm{UU}}=0.32+0.17 /\left[\mathrm{s}_{\mathrm{u}}(\mathrm{UU}) / \mathrm{p}_{\mathrm{a}}\right]$ & 104 & 0.43 & 0.14 \\
\hline & Uplift & $\alpha_{\mathrm{UU}}=0.30+0.17 /\left[\mathrm{s}_{\mathrm{u}}(\mathrm{UU}) / \mathrm{p}_{\mathrm{a}}\right]$ & 44 & 0.69 & 0.15 \\
\hline & All data & $\alpha_{U U}=0.32+0.16 /\left[\mathrm{s}_{\mathrm{u}}(\mathrm{UU}) / \mathrm{p}_{\mathrm{a}}\right]$ & 148 & 0.56 & 0.14 \\
\hline \multirow{3}{*}{$\mathrm{UC}$} & Compression & $\alpha_{\mathrm{UC}}=0.34+0.17 /\left[\mathrm{s}_{\mathrm{u}}(\mathrm{UC}) / \mathrm{p}_{\mathrm{a}}\right]$ & 104 & 0.42 & 0.15 \\
\hline & Uplift & $\alpha_{\mathrm{UC}}=0.30+0.17 /\left[\mathrm{s}_{\mathrm{u}}(\mathrm{UC}) / \mathrm{p}_{\mathrm{a}}\right]$ & 44 & 0.57 & 0.16 \\
\hline & All data & $\alpha_{U C}=0.34+0.16 /\left[\mathrm{s}_{\mathrm{u}}(\mathrm{UC}) / \mathrm{p}_{\mathrm{a}}\right]$ & 148 & 0.56 & 0.15 \\
\hline
\end{tabular}

test [denoted $\mathrm{s}_{\mathrm{u}}(\mathrm{CIUC})$ ] is selected as the appropriate reference test, because it is quite common and of good quality test. To obtain $\mathrm{s}_{\mathrm{u}}$ (CIUC), $\mathrm{s}_{\mathrm{u}}$ values from all other test types are converted to "equivalent" $\mathrm{s}_{\mathrm{u}}(\mathrm{CIUC})$ values. The procedures to convert are based on the conclusions from a research study done by Chen and Kulhawy [3] for unconsolidated-undrained triaxial (UU) and unconfined compression (UC) tests. They developed the strength interrelationships using theoretical base and laboratory test data from all over the world, so these correlations can be applied to general cohesive soils. The representative equations are as follows:

$$
\begin{aligned}
& \mathrm{s}_{\mathrm{u}}(\mathrm{UU}) / \mathrm{s}_{\mathrm{u}}(\mathrm{CIUC})=0.911+0.499 \log \left[\mathrm{s}_{\mathrm{u}}(\mathrm{UU}) / \bar{\sigma}_{\mathrm{vo}}\right] \\
& \mathrm{s}_{\mathrm{u}}(\mathrm{UC}) / \mathrm{s}_{\mathrm{u}}(\mathrm{CIUC})=0.893+0.513 \log \left[\mathrm{s}_{\mathrm{u}}(\mathrm{UC}) / \bar{\sigma}_{\mathrm{vo}}\right]
\end{aligned}
$$

For consolidated-anisotropically undrained triaxial, plane strain, and direct simple shear tests, Kulhawy and Mayne [8] established the equation as follows:

$$
\mathrm{s}_{\mathrm{u}}(\mathrm{CT}) / \bar{\sigma}_{\mathrm{vo}}=\mathrm{a}_{\mathrm{TEST}} \mathrm{a}_{\mathrm{RATE}} \mathrm{a}_{\mathrm{OCR}} \mathrm{s}_{\mathrm{u}}(\mathrm{CIUC}) / \bar{\sigma}_{\mathrm{vo}}
$$

in which $\bar{\sigma}_{v o}=$ effective vertical overburden stress, $s_{u}(C T)=s_{u}$ for converted test type, and $\mathrm{a}=$ coefficients of correction factors for test type, strain rate during testing, and OCR.

Using the above analytical procedures, Fig. 3 illustrates the updated undrained shear strength correlation producing a regression equation as follows:

$$
\begin{aligned}
& \alpha_{\mathrm{CIUC}}=0.30+0.17 /\left[\mathrm{s}_{\mathrm{u}}(\mathrm{CIUC}) / \mathrm{p}_{\mathrm{a}}\right] \\
& \left(\mathrm{n}=148, \mathrm{r}^{2}=0.66, \text { and } \mathrm{SD}=0.09\right)
\end{aligned}
$$

where $s_{u}$ is normalized by one atmospheric stress $\left(p_{a}=101.3\right.$ $\mathrm{kN} / \mathrm{m}^{2}$ ). It is apparent that the data distribution of updated $\alpha-\mathrm{s}_{\mathrm{u}}$ correlation is superior to the previous result in Fig. 1.

Although the $\alpha_{\mathrm{CIUC}}-\mathrm{S}_{\mathrm{u}}$ (CIUC) equation in Fig. 3 is developed using regression analysis, it can be clearly observed that the $\alpha$ value in the regression line is conservative for

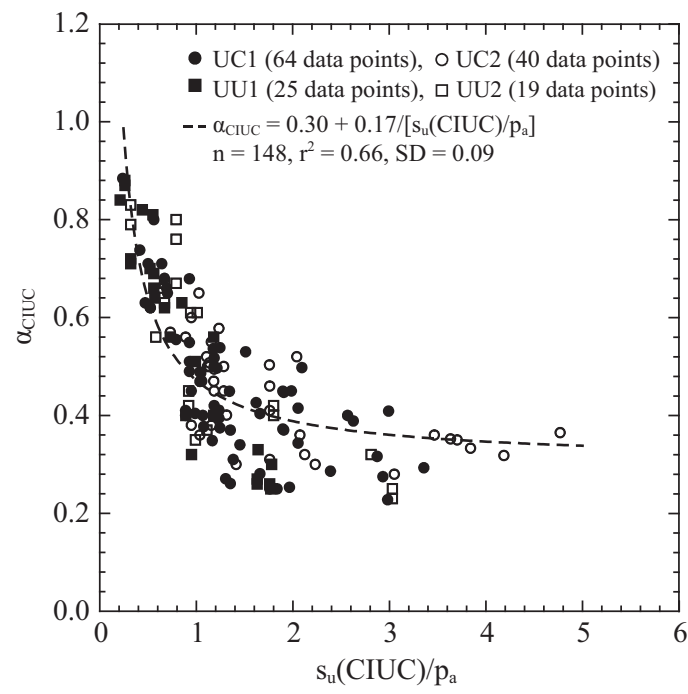

Fig. 3. $\alpha_{\mathrm{CIUC}}-\mathrm{s}_{\mathrm{u}}(\mathrm{CIUC}) / \mathrm{p}_{\mathrm{a}}$ correlation.

$\mathrm{s}_{\mathrm{u}}(\mathrm{CIUC}) / \mathrm{p}_{\mathrm{a}}<1$, since most data points are seen above the regression line. In addition, Fig. 3 also shows that the $\alpha$ value presents a considerably steep slope in small value of $\mathrm{s}_{\mathrm{u}}(\mathrm{CIUC}) / \mathrm{p}_{\mathrm{a}}$. Therefore, the use of $\alpha_{\mathrm{CIUC}}-\mathrm{s}_{\mathrm{u}}(\mathrm{CIUC})$ correlation for design may tend to be conservative and sensitive in the area of smaller $s_{u}$.

Table 6 lists the comparisons of different loadings and test types of undrained shear strength. Comparing uplift and compression, the results show that the values are quite similar. Although a small difference exists, it is not significant enough to warrant differentiation. For convenience, both UU test, denoted $\alpha_{U U}$ versus $s_{u}(U U)$, and UC test, denoted $\alpha_{U C}$ versus $\mathrm{s}_{\mathrm{u}}(\mathrm{UC})$, are also shown in Table 6. As can be seen, $\alpha_{\mathrm{UU}}$ and $\alpha_{U C}$ are somehow greater than $\alpha_{\text {CIUC }}$ because the test result from $\mathrm{s}_{\mathrm{u}}\left(\right.$ CIUC) is typically greater than $\mathrm{s}_{\mathrm{u}}(\mathrm{UU})$ or $\mathrm{s}_{\mathrm{u}}(\mathrm{UC})$ for general clays [3]. Furthermore, the $\alpha$ values from the UU or UC test present larger standard deviations (SD) and are less reliable than those from the CIUC test. This observation is consistent with the common supposition since the quality of the CIUC test is generally better than UU and UC tests. 
Table 7. Summary of statistical data for Fig. 4.

\begin{tabular}{cccc}
\hline \multirow{2}{*}{ USR } & \multicolumn{3}{c}{ Statistics } \\
\cline { 2 - 4 } & $\mathrm{n}$ & $\mathrm{r}^{2}$ & $\mathrm{SD}$ \\
\hline$<1.0$ & 41 & 0.69 & 0.09 \\
$1.0-2.0$ & 39 & 0.56 & 0.09 \\
$2.0-3.0$ & 39 & 0.71 & 0.08 \\
$3.0-4.0$ & 13 & 0.63 & 0.08 \\
$4.0-5.0$ & 14 & 0.30 & 0.09 \\
$>5.0$ & 18 & 0.29 & 0.13 \\
\hline
\end{tabular}

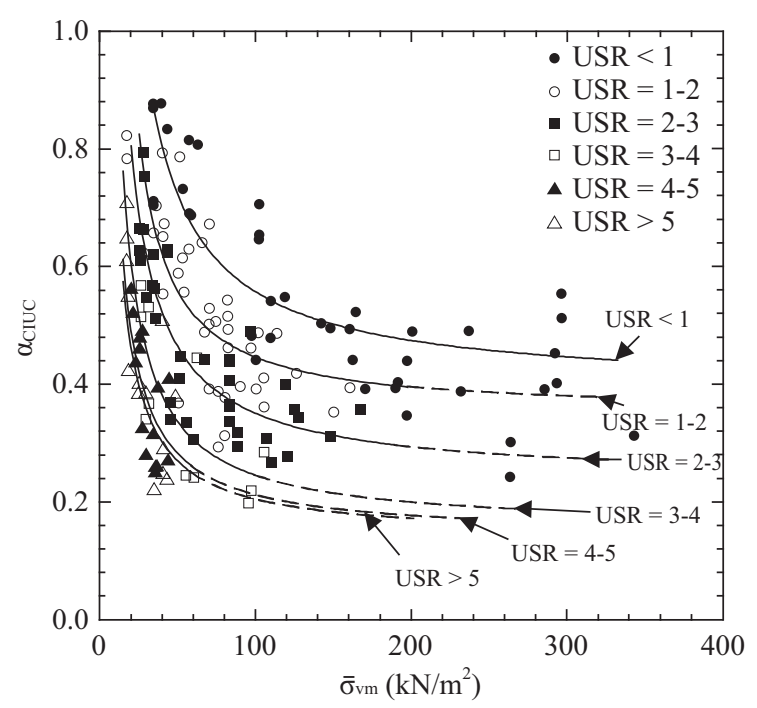

Fig. 4. $\boldsymbol{\alpha}_{\mathrm{CIUC}}-\mathbf{s}_{\mathrm{u}}(\mathrm{CIUC}) / \bar{\sigma}_{\mathrm{vm}}-\bar{\sigma}_{\mathrm{vm}}$ correlations.

\section{2) $\alpha-s_{u} / \bar{\sigma}_{v m}-\bar{\sigma}_{v m}$ Correlations}

Fig. 4 shows the results of undrained strength ratio correlation, $\alpha_{\mathrm{CIUC}}-\mathrm{s}_{\mathrm{u}}(\mathrm{CIUC}) / \bar{\sigma}_{\mathrm{vm}}-\bar{\sigma}_{\mathrm{vm}}$. Meanwhile, Table 7 lists the statistical data for all individual curves in Fig. 4. These correlations are developed directly from the field load test database. Several points can be observed from Fig. 4 and Table 7. First, the coefficients of determination $r^{2}$ are large when USRs are small. The values of $r^{2}$ become smaller when USR is greater than 4, this may be due to the limited data distribution in the $\alpha_{\mathrm{CIUC}}-\mathrm{S}_{\mathrm{u}}(\mathrm{CIUC}) / \bar{\sigma}_{\mathrm{vm}}-\bar{\sigma}_{\mathrm{vm}}$ correlation. Second, the difference of regression lines is minimal when USR is greater than 3 . Furthermore, the regression lines are very close when USR is greater than 4 . Third, the trend of $\alpha_{\text {CIUC }}$ decreases with increasing $\mathrm{s}_{\mathrm{u}}(\mathrm{CIUC}) / \bar{\sigma}_{\mathrm{vm}}$ and $\bar{\sigma}_{\mathrm{vm}}$. In addition, it can be noted that these data points present more consistent results for small $\bar{\sigma}_{\mathrm{vm}}$. On the other hand, the data points are scattered or lacking when the value of $\bar{\sigma}_{\mathrm{vm}}$ increases. By visual observation, some regression lines are also somehow conservative for small $\bar{\sigma}_{\mathrm{vm}}$. The phenomenon is comparable with the result in Fig. 3.
Table 8. Statistical results of back-calculated $K / K_{0}$.

\begin{tabular}{cccccc}
\hline Mode $^{\mathrm{a}}$ & Construction method & $\mathrm{n}$ & Mean & SD & COV \\
\hline \multirow{3}{*}{$\mathrm{D}$} & slurry & 12 & 0.73 & 0.24 & 0.34 \\
& casing & 10 & 0.97 & 0.12 & 0.12 \\
& dry & 52 & 1.03 & 0.36 & 0.35 \\
\hline \multirow{3}{*}{$\mathrm{U}$} & slurry & 16 & 0.79 & 0.28 & 0.35 \\
& casing & 25 & 0.88 & 0.31 & 0.35 \\
& dry & 63 & 1.12 & 0.29 & 0.26
\end{tabular}

$\overline{\mathrm{D}}=$ drained; $\mathrm{U}=$ undrained.

The $\alpha_{\mathrm{CIUC}^{-}} \mathrm{S}_{\mathrm{u}}(\mathrm{CIUC}) / \bar{\sigma}_{\mathrm{vm}}-\bar{\sigma}_{\mathrm{vm}}$ correlations can be regarded as an alternative analysis for traditional $\alpha-s_{u}$ correlations, especially in the condition of having a small $\mathrm{s}_{\mathrm{u}}$. The $\alpha$ value on the $\alpha_{\mathrm{CIUC}}-\mathrm{S}_{\mathrm{u}}(\mathrm{CIUC}) / \bar{\sigma}_{\mathrm{vm}}-\bar{\sigma}_{\mathrm{vm}}$ correlations is more precisely distinguished than using the conventional $\alpha_{\mathrm{CIUC}^{-}} \mathrm{s}_{\mathrm{u}}(\mathrm{CIUC})$ correlations. Therefore, the required $\alpha$ value can be more reasonably selected. It can be seen from the distribution of data in Fig. 4 and Table 7, that the suggested ranges of alternative values are (1) $\bar{\sigma}_{\mathrm{vm}}<200 \mathrm{kN} / \mathrm{m}^{2}$ for USR $<3$ and (2) $\bar{\sigma}_{\mathrm{vm}}<100 \mathrm{kN} / \mathrm{m}^{2}$ for USR $>3$, because the $\alpha_{\mathrm{CIUC}}-\mathrm{S}_{\mathrm{u}}(\mathrm{CIUC}) /$ $\bar{\sigma}_{\mathrm{vm}}-\bar{\sigma}_{\mathrm{vm}}$ correlations in these ranges have shown better statistical results.

\section{2. $\beta$ Method}

\section{1) Drained Load Tests}

The approximate $\beta\left(\beta_{p}\right)$ can be predicted from Eq. (2) and given as:

$$
\beta_{\mathrm{p}}=\mathrm{K}_{\mathrm{o}}\left(\mathrm{K} / \mathrm{K}_{\mathrm{o}}\right) \tan (\bar{\phi} \cdot \delta / \bar{\phi})
$$

Meanwhile, the measured $\beta\left(\beta_{\mathrm{m}}\right)$ is also back-calculated from the field load test results using Eq. (2), as follows:

$$
\beta_{\mathrm{m}}=\mathrm{Q}_{\mathrm{s}}\left(\mathrm{L}_{2}\right) /\left[\pi \mathrm{B} \mathrm{D} \bar{\sigma}_{\mathrm{vm}}\right]
$$

where $\mathrm{K}=$ the operative horizontal stress coefficient, which depends on the original in-situ coefficient of horizontal soil stress $\left(\mathrm{K}_{\mathrm{o}}\right)$, and the other terms have been defined previously. With assumptions of $\beta_{\mathrm{m}}=\beta_{\mathrm{p}}$ and $\delta / \bar{\phi}=1$ for drilled shafts, the stress factor $\mathrm{K} / \mathrm{K}_{\mathrm{o}}$ for overall foundation depth can be backcalculated from Eqs. (9) and (10). Table 8 shows the statistical results of $\mathrm{K} / \mathrm{K}_{\mathrm{o}}$ for the different construction methods. For drained tests, the mean values of $\mathrm{K} / \mathrm{K}_{\mathrm{o}}$ are $0.73,0.97$, and 1.03 for slurry, casing, and dry construction, respectively. The results are obviously larger than in the previous study by Kulhawy et al. [9], which showed $\mathrm{K} / \mathrm{K}_{\mathrm{o}}=2 / 3$ for slurry, 1 for dry construction, and intermediate values between these ranges for casing construction under water.

Fig. 5 shows the comparison of measured and predicted drained $\beta$ based on the suggested $\mathrm{K} / \mathrm{K}_{\mathrm{o}}$ values of this study and 
Table 9. Comparison of $\beta$ analysis using various $K / K_{0}$ suggestions.

\begin{tabular}{cccc}
\hline Reseachers $^{\mathrm{a}}$ & Mode $^{\mathrm{b}}$ & Regression analysis & Mean analysis \\
\hline Kulhawy et al. & $\mathrm{D}$ & $\beta_{\mathrm{m}}=1.13 \beta_{\mathrm{p}}\left(\mathrm{n}=71, \mathrm{r}^{2}=0.95, \mathrm{SD}=0.20\right)$ & Mean $\beta_{\mathrm{m}} / \beta_{\mathrm{p}}=1.13(\mathrm{n}=74, \mathrm{SD}=0.26, \mathrm{COV}=0.23)$ \\
This study & $\mathrm{D}$ & $\beta_{\mathrm{m}}=1.01 \beta_{\mathrm{p}}\left(\mathrm{n}=71, \mathrm{r}^{2}=0.95, \mathrm{SD}=0.21\right)$ & Mean $\beta_{\mathrm{m}} / \beta_{\mathrm{p}}=1.03(\mathrm{n}=74, \mathrm{SD}=0.24, \mathrm{COV}=0.24)$ \\
\hline Kulhawy et al. & $\mathrm{U}$ & $\beta_{\mathrm{m}}=1.19 \beta_{\mathrm{p}}\left(\mathrm{n}=104, \mathrm{r}^{2}=0.96, \mathrm{SD}=0.25\right)$ & Mean $\beta_{\mathrm{m}} / \beta_{\mathrm{p}}=1.13(\mathrm{n}=104, \mathrm{SD}=0.27, \mathrm{COV}=0.24)$ \\
This study & $\mathrm{U}$ & $\beta_{\mathrm{m}}=1.00 \beta_{\mathrm{p}}\left(\mathrm{n}=104, \mathrm{r}^{2}=0.95, \mathrm{SD}=0.29\right)$ & Mean $\beta_{\mathrm{m}} / \beta_{\mathrm{p}}=1.01(\mathrm{n}=104, \mathrm{SD}=0.27, \mathrm{COV}=0.26)$ \\
\hline
\end{tabular}

${ }^{a}$ Kulhawy et al. [9].

${ }^{\mathrm{b}} \mathrm{D}=$ drained; $\mathrm{U}=$ undrained.

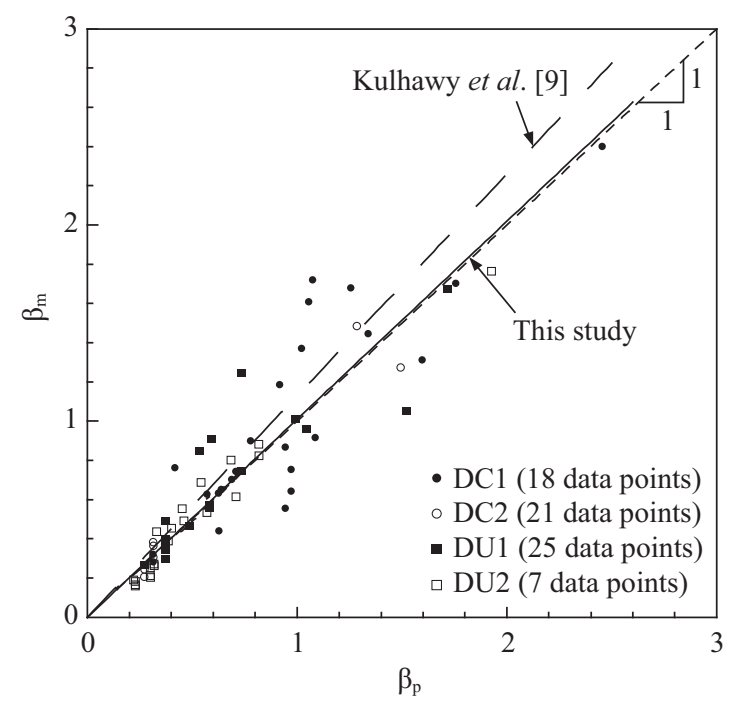

Fig. 5. Comparison of measured and predicted drained $\beta$.

previous research works. Table 9 summarizes the statistical data for both regression and mean analyses. It can be seen in Fig. 5 and Table 9, that the overall measured and predicted results of this study are more consistent than the previous study. From the result of mean $\beta_{\mathrm{m}} / \beta_{\mathrm{p}}=1.13$, it is clear that the suggested $\mathrm{K} / \mathrm{K}_{\mathrm{o}}$ values by Kulhawy et al. [9] are underestimated. In this study, the predicted and measured drained $\beta$ values are much closer. Therefore, the updated analysis for $\mathrm{K} / \mathrm{K}_{\mathrm{o}}$ is suggested because it has shown superiority over the previous study.

The relation of drained $\beta_{\mathrm{m}} / \beta_{\mathrm{p}}$ versus depth is presented in Fig. 6. It can be seen that there is a wide range of $\beta_{\mathrm{m}} / \beta_{\mathrm{p}}$ from 0.5 to 1.9 at shallow depths $(\mathrm{D}<20 \mathrm{~m})$; however, the data distribution becomes narrow (0.7-1.2) when shaft length is greater than $20 \mathrm{~m}$. On average, for shaft length $<20 \mathrm{~m}$, mean $\beta_{\mathrm{m}} / \beta_{\mathrm{p}}=1.06$ with $\mathrm{n}=59, \mathrm{SD}=0.25$, and $\mathrm{COV}=0.24$, while for shafts longer than $20 \mathrm{~m}$, mean $\beta_{\mathrm{m}} / \beta_{\mathrm{p}}=0.91$ with $\mathrm{n}=15$, $\mathrm{SD}=0.16$, and $\mathrm{COV}=0.18$. Therefore, it can be observed that the predicted drained $\beta$ method may be more reliable for shafts greater than $20 \mathrm{~m}$ in length based on these available load test case histories.

\section{2) Undrained Load Tests}

A similar evaluation is done for undrained $\beta$ analysis. Table

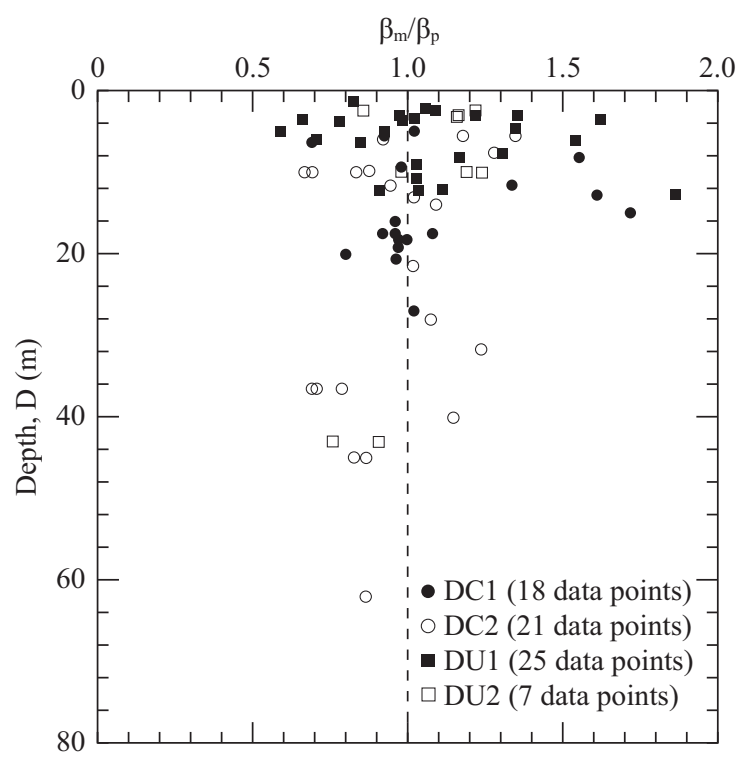

Fig. 6. Drained $\beta_{\mathrm{m}} / \beta_{\mathrm{p}}$ versus depth.

8 also shows the statistical results of back-calculated $\mathrm{K} / \mathrm{K}_{\mathrm{o}}$ for the different construction methods of the undrained tests. The mean values of $\mathrm{K} / \mathrm{K}_{\mathrm{o}}$ are $0.79,0.88$, and 1.12 for slurry, casing, and dry construction, respectively. These results are larger than those in the previous study [9].

Table 9 also summarizes the statistics using different suggested values of $\mathrm{K} / \mathrm{K}_{\mathrm{o}}$ for the undrained tests. It can be seen that the measured and predicted results from this study present more consistent results as well. The reasons for the improvement in the prediction are the same as in the drained tests. Figs. 7 and 8 present the results of the comparison of the measured and predicted $\beta$ and the relation of undrained $\beta_{\mathrm{m}} / \beta_{\mathrm{p}}$ versus depth, respectively. On average, the mean undrained $\beta_{\mathrm{m}} / \beta_{\mathrm{p}}$ is 1.01 and $\beta_{\mathrm{m}}=1.00 \beta_{\mathrm{p}}$ for the regression analysis. The average predicted undrained $\beta$ is consistent with the measured undrained $\beta$.

The relation of undrained $\beta_{\mathrm{m}} / \beta_{\mathrm{p}}$ versus depth also presents a wide range of values $(0.6-1.8)$ at shallow depths $(\mathrm{D}<20 \mathrm{~m})$, but the data distribution becomes narrow $(0.7-1.3)$ when shaft length is greater than $20 \mathrm{~m}$. On average, for short shafts, mean $\beta_{\mathrm{m}} / \beta_{\mathrm{p}}=1.02$ with $\mathrm{n}=78, \mathrm{SD}=0.29$, and $\mathrm{COV}=0.29$, while for long shafts, mean $\beta_{\mathrm{m}} / \beta_{\mathrm{p}}=0.98$ with $\mathrm{n}=26, \mathrm{SD}=0.17$, and $\mathrm{COV}=0.17$. Therefore, as in drained tests, the predicted 


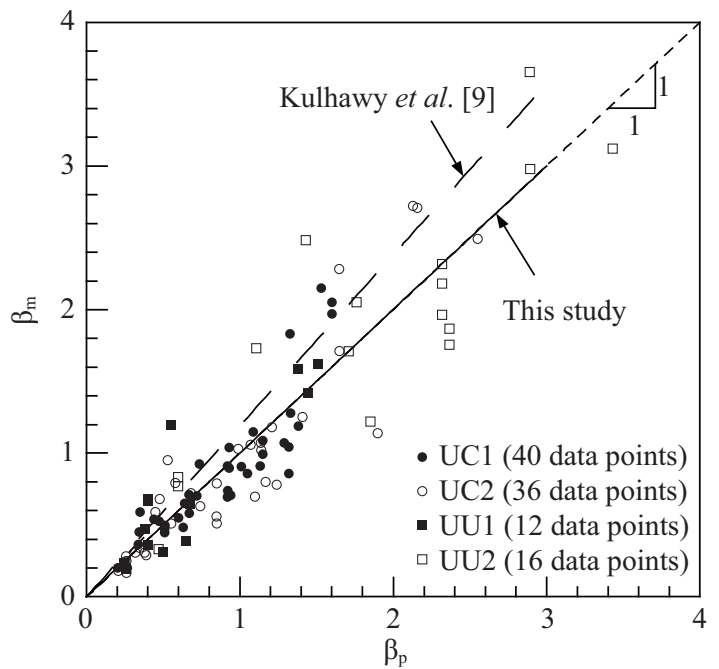

Fig. 7. Comparison of measured and predicted undrained $\beta$.

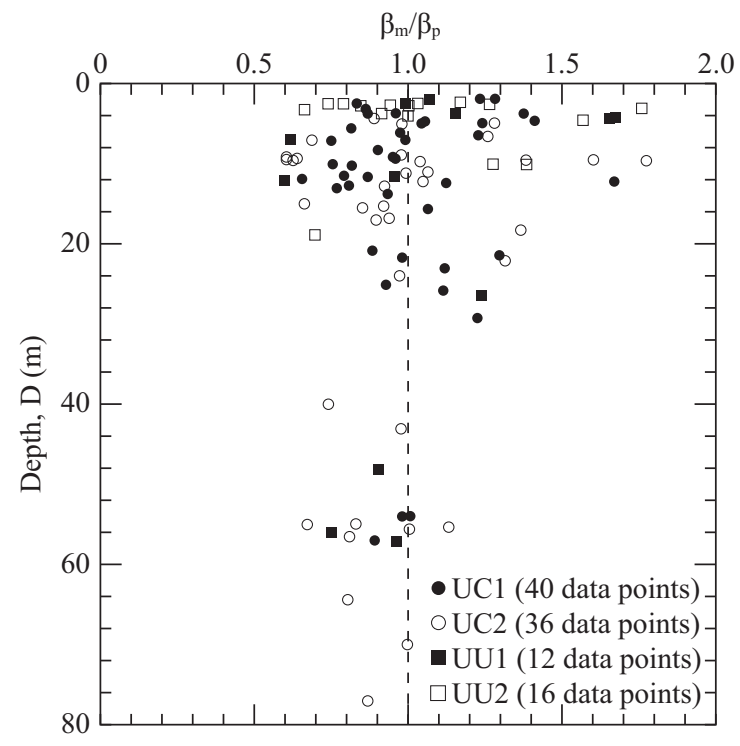

Fig. 8. Undrained $\beta_{\mathrm{m}} / \beta_{\mathrm{p}}$ versus depth.

undrained $\beta$ method is also somehow more reliable for shafts greater than $20 \mathrm{~m}$ in length based on these available load test case histories.

\section{3. $\lambda$ Method}

The measured $\lambda$ can be computed from Eq. (3) and given as:

$$
\lambda=\mathrm{Q}_{\mathrm{s}}\left(\mathrm{L}_{2}\right) /\left[\pi \mathrm{B} \mathrm{D}\left(\bar{\sigma}_{\mathrm{vm}}+2 \mathrm{~s}_{\mathrm{u}}\right)\right]
$$

in which all terms have been defined previously. Fig. 9 shows the results of $\lambda$ versus shaft depth. It can be seen that the range of data points is very wide $(0.05-0.35)$ for short shafts $(\mathrm{D}<30$ $\mathrm{m})$ with mean $\lambda=0.19, \mathrm{n}=131, \mathrm{SD}=0.07$, and $\mathrm{COV}=0.36$. For long shafts, $(\mathrm{D}>30 \mathrm{~m})$, the statistical results are mean $\lambda=$ $0.11, \mathrm{n}=17, \mathrm{SD}=0.02$, and $\mathrm{COV}=0.20$. Based on the

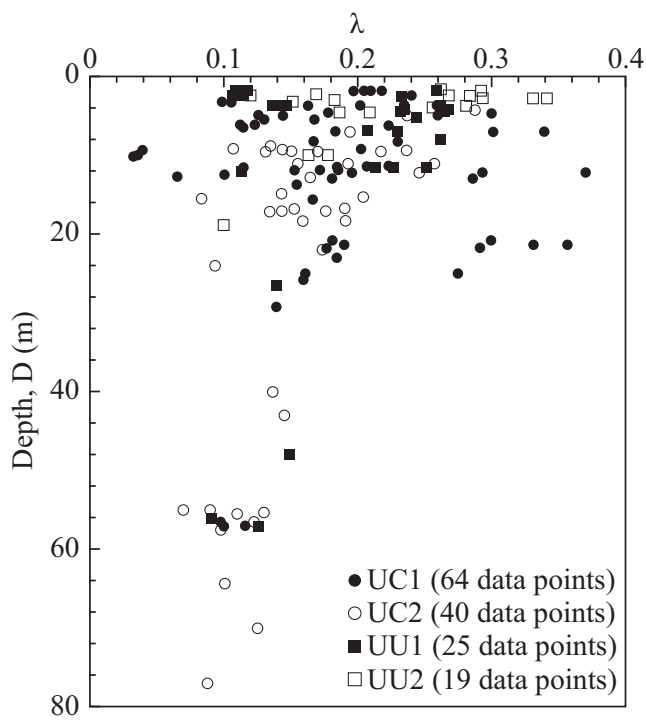

Fig. 9. $\lambda$ versus depth.

available data points, the $\lambda$ value seems convergent to a consistent value when the shaft depth is greater than $30 \mathrm{~m}$. However, more load tests data are needed to examine whether this phenomenon is a significant issue or just a database issue. According to these statistics, it is obvious that the COV of the $\lambda$ method is larger than that of the $\beta$ method. Therefore, the prediction of side resistance of the $\lambda$ method is generally less reliable than $\alpha$ and $\beta$ methods.

\section{DESIGN RECOMMENDATIONS}

For undrained loading, both $\alpha$ and $\beta$ methods present more reasonable results based on the data analyses. The traditional $\alpha-s_{u}$ correlations in Table 6 are proposed for the prediction of undrained side resistance. The new $\alpha_{\mathrm{CIUC}}-\mathrm{s}_{\mathrm{u}}(\mathrm{CIUC}) / \bar{\sigma}_{\mathrm{vm}}-\bar{\sigma}_{\mathrm{vm}}$ correlations can be used as an alternative analytical method, especially in the case of smaller $\mathrm{s}_{\mathrm{u}}$. For $\beta$ analysis, the suggested stress factors $\mathrm{K} / \mathrm{K}_{0}$ are $0.79,0.88$, and 1.12 for slurry, casing, and dry construction, respectively, but the predicted $\beta$ method presents somehow to be more variable for short shafts. However, $\alpha$ method has a more favorable data distribution along the regression line. The prediction of side resistance of the $\lambda$ method is generally less reliable than $\alpha$ and $\beta$ methods.

For drained loading, the analysis of the $\beta$ method is reasonable and the suggested values of $\mathrm{K} / \mathrm{K}_{\mathrm{o}}$ are $0.73,0.97$, and 1.03 for slurry, casing, and dry construction, respectively. However, the predicted $\beta$ method presents somehow to be less reliable for short shafts.

\section{V.SUMMARY AND CONCLUSIONS}

A wide variety of load test data were used for the evaluation of side resistance of drilled shafts under axial loading. Representative analytical models were examined in detail using 
both measured data and predicted results. Based on the evaluation, the following design recommendations for engineering practice are proposed.

(1) For undrained loading, the total stress analysis method, $\alpha_{\text {CIUC }^{-}} \mathrm{S}_{\mathrm{u}}(\mathrm{CIUC})$ correlation, presents more reliable results.

(2) The new correlation, $\alpha_{\mathrm{CIUC}}-\mathrm{s}_{\mathrm{u}}(\mathrm{CIUC}) / \bar{\sigma}_{\mathrm{vm}}-\bar{\sigma}_{\mathrm{vm}}$, which is developed using field load test data, can be regarded as an alternative analysis method for the drilled shaft design, especially in the case of smaller $\mathrm{s}_{\mathrm{u}}$.

(3) For drained loading, the effective stress $\beta$ analysis is a suitable method. Based on the analyses of the available database, it is reasonably consistent for long shafts, but has a wide range of results for short shafts. A similar situation is found for undrained loading.

(4) The suggested stress factors $K / K_{0}$ can substantially improve the prediction model of $\beta$ analysis. For drained loading, the suggested values of $\mathrm{K} / \mathrm{K}_{\mathrm{o}}$ are 0.73 , 0.97 , and 1.03 for slurry, casing, and dry construction, respectively; for undrained loading, the suggested values of $\mathrm{K} / \mathrm{K}_{\mathrm{o}}$ are $0.79,0.88$, and 1.12 for slurry, casing, and dry construction, respectively.

(5) The range of the $\lambda$ method is wide and COV is large for the analysis of side resistance. Thus, the $\lambda$ method for cohesive soils presents less reliable results.

\section{ACKNOWLEDGMENTS}

This study was supported by the National Science Council, Taiwan, R.O.C., under contract number: NSC 97-2221-E-033044-MY2.

\section{REFERENCES}

1. Chen, Y. J., Chang, H. W., and Kulhawy, F. H., "Evaluation of uplift interpretation criteria for drilled shaft capacity," Journal of Geotechnical and Geoenvironmental Engineering, ASCE, Vol. 134, No. 10, pp.
1459-1468 (2008).

2. Chen, Y. J. and Fang, Y. C., "Critical evaluation of compression interpretation criteria for drilled shafts," Journal of Geotechnical and Geoenvironmental Engineering, ASCE, Vol. 135, No. 8, pp. 1056-1069 (2009).

3. Chen, Y. J. and Kulhawy, F. H., "Undrained strength interrelationships among CIUC, UU and UC tests," Journal of Geotechnical Engineering, ASCE, Vol. 119, No. 11, pp. 1732-1750 (1993).

4. Goh, A. T. C., Kulhawy, F. H., and Chua, C. G., "Bayesian neural network analysis of undrained side resistance of drilled shafts," Journal of Geotechnical Engineering, ASCE, Vol. 131, No. 1, pp. 4-93 (2005).

5. Hirany, A. and Kulhawy, F. H., "Conduct and interpretation of load tests on drilled shaft foundations: Detailed guidelines," Report EL-5915, Vol. 1, EPRI, Palo Alto (1988).

6. Hirany, A. and Kulhawy, F. H., "On the interpretation of drilled foundation load test results," In: O'Neill, M. W. and Townsend, F. C. (Eds.), Deep Foundations 2002 (GSP 116), ASCE, Reston, pp. 1018-1028 (2002).

7. Kulhawy, F. H. and Jackson, C. S., "Some observations on undrained side resistance of drilled shafts," Proceeding of the Foundation Engineering: Current Principles and Practices, ASCE, New York, pp. 1011-1025 (1989).

8. Kulhawy, F. H. and Mayne, P. W., "Manual on estimating soil properties for foundation design," Report EL-6800, EPRI, Palo Alto (1990).

9. Kulhawy, F. H., Trautmann, C. H., Beech, J. F., O`Rourke, T. D., McGuire, W., Wood, W. A., and Capano, C., "Transmission line structure foundations for uplift-compression loading," Report EL-2870, EPRI, Palo Alto (1983).

10. Mayne, P. W. and Kulhawy, F. H., "K $\mathrm{K}_{\mathrm{O}} \mathrm{OCR}$ relationships in soils," Journal of Geotechnical Engineering, ASCE, Vol. 108, No. GT6, pp. 851-872 (1982).

11. Randolph, M. F. and Murphy, B. S., "Shaft capacity of driven piles in clay," Proceeding of the 17th Offshore Technical Conference, Houston, Texas, Vol. 1, pp. 371-378 (1985).

12. Semple, R. M. and Rigden, W. J., "Shaft capacity of driven pipe piles in clay," Ground Engineering, Vol. 19, No. 1, pp. 11-17 (1986).

13. Stas, C. V. and Kulhawy, F. H., "Critical evaluation of design methods for foundations under axial uplift and compression loading," Report EL-3771, EPRI, Palo Alto (1984).

14. Tomlinson, M. J., "The adhesion of piles driven in clay soils," Proceeding of the 4th International Conference on Soil Mechanics and Foundation Engineering, London, Vol. 2, pp. 66-71 (1957).

15. Vijayvergiya, V. N. and Focht, J. A., "A new way to predict capacity of piles in clay," Proceeding of the 4th Offshore Technology Conference, Houston, Texas, Vol. 2, pp. 865-874 (1972). 